\title{
Communication versus (Restricted) Delegation: An Experimental Comparison
}

Silvia Dominguez Martinez Randolph Sloof Netherlands. 
Tinbergen Institute is the graduate school and research institute in economics of Erasmus University Rotterdam, the University of Amsterdam and VU University Amsterdam.

More TI discussion papers can be downloaded at http://www.tinbergen.nl

Tinbergen Institute has two locations:

Tinbergen Institute Amsterdam

Gustav Mahlerplein 117

1082 MS Amsterdam

The Netherlands

Tel.: +31(0)20525 1600

Tinbergen Institute Rotterdam

Burg. Oudlaan 50

3062 PA Rotterdam

The Netherlands

Tel.: +31(0)10 4088900

Fax: +31(0)10 4089031 


\title{
Communication versus (restricted) delegation: An experimental comparison*
}

\author{
Silvia Dominguez Martinez ${ }^{a}$ and Randolph Sloof ${ }^{a, b}$ \\ ${ }^{a}$ University of Amsterdam and the Tinbergen Institute \\ ${ }^{b}$ Copenhagen Business School
}

July 2016

\begin{abstract}
This paper reports the results from a laboratory experiment investigating a manager's decision whether or not to delegate authority to a better informed worker whose interests are often, but not always, congruent. Keeping authority implies a loss of information, as the worker communicates his information strategically. Delegating authority leads to a loss of control. A key aspect of our design is that the manager can restrict the worker's choice set when delegating authority. We find that, in case of delegation, managers (as predicted) put tighter restrictions when interests are less aligned. Workers send more informative messages under communication than predicted by the pure strategy equilibria. This finding neither appears to be driven by lying aversion of workers nor by credulity of managers. Qualitatively, our results are in line with a mixed strategy equilibrium under communication, which strictly outperforms optimal restricted delegation and is relatively close to the optimal stochastic mechanism in our setting.
\end{abstract}

JEL: C90, D80, M20.

Keywords: Delegation, Communication, Laboratory Experiment, Organizational Economics.

\footnotetext{
${ }^{*}$ We thank the priority research area Behavioral Economics of the University of Amsterdam for financial support. Corresponding author: S.DominguezMartinez@uva.nl.
} 


\section{Introduction}

Apart from the performance evaluation and reward system, a key feature of organizational architecture is the assignment of decision rights. In deciding whether to delegate decision authority to lower level employees (and to what extent), managers face a basic trade-off between a loss of control and a loss of information (cf. Jensen and Meckling, 1992). Lower level employees are typically better informed and therefore able to take more informed decisions. At the same time, they may have different interests. Providing them with decision making power thus potentially leads to different decisions than the manager would have taken if she would have the same information. This loss of control under delegation has to be compared with the loss of information under centralized decision making. Even though manager and agent can communicate under centralization, the divergent interests imply that agents do so strategically. This typically precludes full information disclosure, such that the manager's decision will be based on coarse information.

The loss of control versus loss of information trade-off has received widespread theoretical attention. Dessein (2002) for instance shows that, in the cheap talk setting of Crawford and Sobel (1982), full delegation outperforms direct communication (as long as the incentive conflict is not too large relative to the manager's uncertainty about the environment). The intuition here is that the bias in information transmission that results under centralization is an order of magnitude larger than the bias in decision making after delegation. Managers are therefore predicted to delegate authority, even though this leads to a loss of control. In fact, theoretically managers are even better off when they impose an upper limit on the level of the agent's discretion, i.e. opt for 'restricted delegation' (see also Holmstrom, 1984; Ottaviani, 2000; Alonso and Matouschek, 2008). Moreover, restricted delegation implements the second best outcome, i.e. corresponds to the optimal general mechanism (Goltsman et al, 2009; Kováč and Mylovanov, 2009).

The frequent observation of highly centralized organizations suggests that delegation does not always dominate communication. Indeed, Dessein's main result does not generalize to some other, arguably relevant situations. A key assumption in the setup of Dessein is that the agent's bias is common knowledge. If the bias is a priori uncertain, the effectiveness of communication is improved and the trade-off can go either way. Centralization then may outperform (full) delegation (cf. Dessein, 2002, Section 8.2; Ottaviani, 2000; Rush et al., 2010). ${ }^{1}$ Also on behavioral grounds

\footnotetext{
${ }^{1}$ Other key assumptions are that the sender is fully informed on the state and that the receiver has no private information of his own. Otherwise cheap talk may dominate delegation in some cases; see e.g. Agastya et al (2014) for a setting where the sender only observes one dimension of the (essentially two dimensional) state of the world and Garfagnini et al (2014) for a setup where the receiver has additional (private) information.
} 
one may expect that communication performs better than the analysis in Dessein (2002) suggests. Experimental studies of signaling games, for example, typically find that cheap talk is far more informative than standard theory predicts; see e.g. Cai and Wang (2006) and de Haan et al (2015). One plausible explanation put forward is that most people are somewhat lying averse. If agents are sufficiently lying averse, the bias in information transmission under centralization is much lower and communication may become superior to delegation (cf. Kartik, 2009; Proposition 4). Moreover, a behavioral force that may limit the attractiveness of restricted delegation is that most people dislike being restricted and are willing to costly punish the manager when they are (Falk and Kosfeld, 2006). This may lower the actual attractiveness of restricted delegation as compared to standard theoretical predictions.

In this paper we explore the driving forces that govern a manager's delegation choice by means of a laboratory experiment. We focus on a very simple setting, in which the worker's bias varies with the state of the world and thus is uncertain for the manager (cf. Blume et al., 1998, 2001). In three equally likely states preferences are perfectly aligned, in the fourth state preferences are opposed. Depending on the probability $1-q$ that the latter 'conflict' state occurs, full delegation may either be better or worse than communication. Our highly stylized setup thus captures situations where the trade-off can go either way. A key characteristic of our design is that the manager may restrict the agent's choice set when delegating authority. Focussing on pure strategy equilibria, optimal (restricted) delegation then theoretically outperforms optimal communication. Behaviorally, however, we expect that the manager may prefer communication. On the one hand, communication is likely to perform better when agents are lying averse. On the other hand, restricted delegation may perform worse because workers may dislike being restricted.

Our main experimental findings are as follows. If managers delegate, they (as predicted) put tighter restrictions in case interests are less aligned. Workers send more informative messages under communication than predicted by the pure strategy equilibria. This finding neither appears to be driven by lying aversion of workers nor by credulity of managers. Qualitatively, our results are in line with a mixed strategy equilibrium under communication, which strictly outperforms optimal restricted delegation and is relatively close to the optimal stochastic mechanism in our setting. Our results thus tentatively suggest that centralization may be an enforceable and realistic way to reap part of the benefits of stochastic allocation mechanisms, and provide an (standard instrumental) explanation why managers are less inclined to delegate than to keep decision authority.

A large number of experimental studies on delegation already exist. One strand of the literature 
focuses on strategic delegation as commitment device or as a way to shift responsibility. ${ }^{2}$ Closer to our study are experiments that study the informational role of delegation. ${ }^{3}$ Fehr et al (2013), Dominguez-Martinez et al (2014) and Sloof and von Siemens (2015) focus on the motivational consequences of (effective) delegation and examine a setup based on Aghion and Tirole (1997) in which the worker has to exert costly effort to become informed. If the manager keeps authority or closely monitors the worker, the worker has weakened incentives to gather information, leading to a loss of initiative. This has to be traded off against the loss of control in case authority is delegated or the manager does not monitor. Like us, Lai and Lim (2012) also consider a communicationdelegation setting in which the worker is informed from the outset and loss of information under centralization is due to strategic communication. The worker can be of two types and two different strategic situations are studied. In game $\mathrm{C}$ the most preferred action in both states for the worker is the least preferred action of the manager (action $a_{4}$ in their Figure 1). Preferences over the other three actions in this game are perfectly aligned. Communication thus allows full revelation of information while at the same time making sure that $a_{4}$ is not chosen. This makes communication far more attractive for the manager than full delegation. Game D drops action $a_{4}$ and changes the preferences of the worker over the remaining three actions in such a way that in one state preferences are aligned while in the other state they are not. This gives the worker an incentive to always claim that the congruent state applies and strategic communication is predicted to be uninformative. The manager is thus better off delegating. The experimental findings largely confirm these predictions, although in game D there is still a substantial fraction of managers that does not delegate. Lai and Lim (2012) show that this can be explained within a level- $k$ framework, in which under-delegation results due to a belief that a less-than-fully strategic worker will provide some information.

Our experiment differs in a number of respects from Lai and Lim (2012). First and foremost, we allow for restricted delegation as well. This is important from both a theoretical and a practical perspective. Note, for instance, that in game $\mathrm{C}$ of Lai and Lim restricted delegation - with action $a_{4}$ excluded from the worker's choice set - would be fully equivalent to communication. It may thus very well be the case that delegation would have been predominantly chosen if managers could

\footnotetext{
${ }^{2}$ See e.g. Fershtman and Gneezy (2001), Hamman et al (2010), Coffman (2011) and Bartling and Fischbacher (2012). Another strand of the literature focuses on carefully inferring individual's intrinsic valuation of decision authority and control (beyond the instrumental benefits), see e.g. Bartling et al (2014) and Owens et al (2014), thereby providing a rationale why in general people are reluctant to delegate.

${ }^{3}$ Other recent experiments consider settings with one head office and two divisions and focus on the tradeoff between improving coordination between the divisions through centralization versus facilitating adaptation to local circumstances via delegation; see e.g. Brandts and Cooper (2015), Evdokimov and Garfagnini (2015) and Hamman and Martinez-Carrasco (2015).
} 
optimally limit the worker's discretion. Second, we employ a richer message space, allowing the worker to either make a factual statement about the state, or a recommendation about which project to choose. This provides additional insights in how strategic communication actually takes place. Third, instead of a level- $k$ analysis as possible driver of over-communication, we experimentally explore lying aversion by including individual incentivized measures of lying aversion and credulity. Fourth, we provide a comparison with the optimal stochastic mechanism. ${ }^{4}$

The remainder of this paper is organized as follows. Section 2 presents the theoretical predictions. In Section 3 we describe the experimental design. In Section 4 results are presented. Section 5 discusses potential explanations for the findings presented in Section 4. Section 6 concludes.

\section{The model and its predictions}

\subsection{Strategic situation}

We consider a game between a manager and a worker. The manager has the initial decision authority which project $a \in\{A, B, C\}$ to implement. The optimal project choice depends on the state of the world $t \in\{1,2,3,4\}$. Ex ante states 1, 2, and 3 each occur with a probability of $\frac{1}{3} q$ while state 4 occurs with probability $1-q$ (for $0<q<1$ ). The worker knows the actual state of the world, the manager only its prior distribution. In the 'congruent' states 1,2 , and 3 preferences of manager and worker are perfectly aligned; in these states both prefer project $A, B$, and $C$, respectively. In the 'conflict' state 4 preferences are completely opposed. The manager then prefers project $A$ while the worker prefers $C$ (and the in between project $B$ can be seen as a compromise solution). Parameter $q$ can thus be interpreted as a (ex ante) measure of interest alignment (cf. Pitchik and Schotter, 1987). The larger $q$ is, the higher the interest alignment between manager and worker is. ${ }^{5}$

In our experimental implementation of the above strategic situation, payoffs over state-action

\footnotetext{
${ }^{4}$ Another difference is that our two treatments can be unambiguously ordered in terms of interest alignment. When moving from game $\mathrm{C}$ to game D in Lai and Lim (2012), two things change at the same time: (i) action $a_{4}$ is dropped, leading to better aligned incentives, and (ii) changes in the worker's payoffs belonging to action $a_{2}$ are made leading to less aligned incentives. The games are thus not straightforwardly ordered (see also the discussion on p. 544 in Lai and Lim (2012)). Yet in terms of predictions their game C resembles our weak alignment case (communication outperforms full delegation but is equivalent to restricted delegation), while game D resembles our strong alignment treatment (full delegation outperforms communication).

${ }^{5}$ Pitchik and Schotter (1987) study a discrete model of strategic information transmission with two states and two actions. The preferred action of the receiver varies with the state, the preferred action of the sender does not (although the intensity of his preferences do). The state where preferences over actions coincide occurs with probability $r$. Pitchik and Schotter compare their setup with the framework of Crawford and Sobel and argue that $r$ can be interpreted as a measure of (ex ante) interest alignment.
} 
pairs are given by Table 1 below: ${ }^{6}$

Table 1: Payoffs over state-project pairs

\begin{tabular}{rcccc}
\hline \hline $\operatorname{Pr}(t)$ & $t$ & $A$ & $B$ & $C$ \\
\hline$q / 3$ & 1 & 80,80 & 0,0 & 0,0 \\
$q / 3$ & 2 & 0,0 & 100,100 & 0,0 \\
$q / 3$ & 3 & 0,0 & 10,10 & 100,100 \\
$1-q$ & 4 & 120,10 & 80,80 & 10,120 \\
\hline \hline
\end{tabular}

Note: The first payoff belongs to the manager,

the second payoff belongs to the worker.

In the absence of any information, the manager would either choose project $A$ or $B$ depending on the value of $q$ (project $C$ is always dominated by project $B$ in expected payoff terms). Because the manager's preferred project depends on the state, he would like to take a more informed decision. One possibility to do so is full or partial delegation of the decision right to the worker. Another possibility is that the worker sends a cheap talk message about the state before the manager takes a decision ('centralization/communication'). Of these two options, the manager prefers the one that yields him the most in expected payoff terms.

\subsection{Equilibrium predictions}

First consider the delegation case. Let $\Delta \subseteq\{A, B, C\}$ denote the set of projects the manager allows the worker to choose from. A selfish worker then chooses the project from $\Delta$ that yields him the most. Anticipating this, the manager's optimal level of delegation follows from a straightforward comparison of expected payoffs (proofs are relegated to Appendix A):

Proposition 1. The optimal level of delegation for the manager equals:

(a) If $q \geq \frac{7}{10}$, then Full Delegation (referred to as Del-(3)): $\Delta=\{A, B, C\}$;

(b) If $\frac{12}{23} \leq q<\frac{7}{10}$, then Restricted Delegation (Del-(2)): $\Delta=\{A, B\}$;

(c) If $q<\frac{12}{23}$, then No Delegation (Del-(1)): $\Delta=\{A\}$.

\footnotetext{
${ }^{6}$ The general structure of payoffs (i.e. preference order) has been chosen such that (i) under delegation the manager may either consider to exclude no project, to exclude project $C$, or to exclude both $B$ and $C$, whereas (ii) under centralization a worker knowing that $t=4$ always has an incentive to pretend being $t=3$. The exact payoff levels are subsequently chosen to draw the predictions in the next subsection sufficiently far apart.
} 
Proposition 1 is intuitive. If preferences are well aligned ( $q$ high), the manager is best off by delegating full decision power. In case preferences are badly aligned ( $q$ low), the manager should effectively not delegate at all and fully restrict the worker's "choice". When interest alignment is in between ( $q$ intermediate), restricting the worker to choosing either $A$ or $B$ is optimal.

Next consider the centralization/communication case. Here the manager always takes the project decision, after having received a cheap talk message from the worker about the state of the world. Without loss of generality we assume there are four possible messages $m \in\left\{m_{1}, m_{2}, m_{3}, m_{4}\right\}$. For ease of reference, these messages can be interpreted as literal statements about the state. Alternatively, messages $m_{1}, m_{2}$, and $m_{3}$ could be interpreted as saying that the best project for both is $A, B$, or $C$ respectively, and $m_{4}$ that there is a conflict of interests. As is standard in cheap talk games, messages only get their meaning in equilibrium though.

For now we assume that the manager employs a pure strategy. This is partly in keeping with the literature; as Sobel (2013, fn. 7) notes, most applications of sender-receiver games assume that the receiver has a unique best response to each distribution over types, ruling out mixed equilibrium strategies for the receiver by design. In our game the receiver does not have a unique best response to every possible type distribution. We therefore return to considering mixed strategies in Section 5 where we discuss our results. When the manager uses a pure strategy, there is little loss in generality in assuming that the worker types do so as well. The type space can then be partitioned into subsets of types that the manager can distinguish in equilibrium. Proposition 2 characterizes the equilibrium partitions $\Upsilon$ and the corresponding project choices if pure strategies are assumed.

Proposition 2. Assuming that worker and manager employ a pure strategy, the communication game allows the following equilibrium partitions:

(a) For $q \geq \frac{33}{43}$, Communication (Com-(3)): $\Upsilon=\{\{1\},\{2\},\{3,4\}\}$. The corresponding project choices equal $\{A, B, C\}$;

(b) For $q \geq \frac{33}{35}$, Limited Communication $I: \Upsilon=\{\{2\},\{1,3,4\}\}$. The corresponding project choices equal $\{B, C\}$;

(c) For $q \geq \frac{33}{43}$, Limited Communication II: $\Upsilon=\{\{1,2\},\{3,4\}\}$. The corresponding project choices equal $\{B, C\}$;

(d) For $q \geq \frac{12}{23}$, Limited Communication III (Com-(2)): $\Upsilon=\{\{1\},\{2,3,4\}\}$. The corresponding project choices equal $\{A, B\}$; 
(e) For all q, No Communication (Com-(1)): $\Upsilon=\{\{1,2,3,4\}\}$ : The corresponding project choice equals $A$ if $q<\frac{4}{5}$ and $B$ if $q>\frac{4}{5}$.

For higher values of $q$ different communication equilibria exist side by side. In these cases both worker and manager alike prefer the equilibrium in which the larger amount of (influential) information is transmitted, i.e. with the finer partition structure. Given their common interest in this, we therefore assume that the manager and worker communicate 'optimally'; they always coordinate on the most informative communication equilibrium. Note that Limited Communication I and II (parts (b) and (c) in Proposition 2) are never optimal. Because preferences are fully aligned when $t=1$ or $t=2$, both players then strictly prefer equilibrium Com-(3) that exists at the same time. Yet the other three outcomes correspond to optimal communication for at least some values of $q \cdot{ }^{7,8}$

From Proposition 1 and 2 it follows that optimal delegation and optimal communication often lead to the same outcome. If this is not the case, optimal delegation yields the manager more. Proposition 3 summarizes this immediate observation.

Proposition 3. Comparing optimal delegation with optimal communication in terms of expected payoffs for the manager, it holds that:

(a) If $q \geq \frac{33}{43}:$ Del-(3) $=$ Com-(3);

(b) If $\frac{7}{10} \leq q<\frac{33}{43}$ : Del-(3) $>$ Com-(2);

(c) If $\frac{12}{23} \leq q<\frac{7}{10}$ : Del-(2) $=$ Com- $(2)$;

(d) If $q<\frac{12}{23}:$ Del-(1) $=$ Com-(1).

\footnotetext{
${ }^{7}$ Another justification to focus on optimal communication is the neologism proofness refinement of Farrell (1993). In the present context neologisms are (out-of-equilibrium) messages that are assumed to have a literal meaning of the form: "choose project $a$, because my type belongs to set $S$ ". A neologism is credible if: (i) all types in $S$ prefer $a$ over the project they receive in equilibrium, (ii) all types outside $S$ prefer their equilibrium project over $a$ and (iii) choosing $a$ is a best response for the manager when restricting the support of his prior beliefs to $S$. An equilibrium is neologism proof if no credible neologisms exist. Outcomes Limited Communication I and II are not neologism proof, while the other three outcomes in Proposition 2 are neologism proof when $q$ is such that they are the most informative equilibrium available. To see this, note that with $C$ chosen after $m_{\{1,3,4\}}$ in Limited Communication I, type $t=1$ can profitably send the neologism "Choose $A$, because my type belongs to $\{1\}$ ". None of the other types then has an incentive to mimic, while $t=1$ strictly gains. A similar reasoning holds for type $t=1$ in part (c). In outcome Limited Communication III (part (d)) the only neologism that may have a bite is: "choose $C$, because my type belongs to $\{3,4\}$ ". This requires $q \geq \frac{33}{43}$ for $C$ to be a best response. So Com- $(2)$ is not neologism proof if the more informative outcome Com-(3) of part (a) exists. A similar reasoning applies for the Com-(1) outcome when $q>\frac{12}{23}$.

${ }^{8} \mathrm{~A}$ conservative sufficient condition on incentive alignment for effective communication to occur is the partial common interest (PCI) condition of Blume et al. (2001). In our game only the trivial partition $\{1,2,3,4\}$ satisfies PCI, independent of $q$. According to PCI informative communication thus need not necessarily take place.
} 
Proposition 3 reveals that optimal delegation has the advantage that - for intermediate values of $q$-decision making is more sensitive to the information available than strategic communication is. This is reminiscent of Dessein's (2002) finding in the standard Crawford-Sobel framework that delegation outperforms communication if interests are sufficiently aligned such that communication can be influential. It is important to note, however, that Proposition 3 compares optimal delegation with optimal communication. The result does not imply that full delegation is always better than communication, even not when the level of interest alignment is relatively large and communication can be influential. To illustrate, suppose that $\frac{12}{23} \leq q<\frac{7}{10}$. From Proposition 1 it then follows that the manager prefers the restricted delegation outcome to the full delegation outcome, while Proposition 2(c) indicates that the former can be reached under communication. As noted in the Introduction, the improved effectiveness of communication in our setup is partly due to the alignment of interests not being constant across states. Ottaviani (2000), for instance, obtains a similar result when extending the standard Crawford-Sobel framework by assuming that the receiver's bias is a priori uncertain (equal to either $-b$ or $b$ with equal probabilities).

In our experiment we are especially interested in testing whether managers use the option to restrict the worker's choice set in case of delegation, how this varies with the alignment of interests as represented by parameter $q$, and how delegation compares to communication. To that purpose we consider two different levels of interest alignment: $q=\frac{3}{4}$ and $q=\frac{3}{5}$, belonging to parts (b) and (c) of Proposition 3, respectively. Regarding delegation, standard theory then predicts that managers restrict more when interests become less aligned. For the very same drop in interest alignment communication is unaffected though. And comparing (optimal) delegation with communication,

delegation performs strictly better under strong interest alignment $\left(q=\frac{3}{4}\right)$, yet this comparative advantage vanishes when $q$ drops to $q=\frac{3}{5}$.

\subsection{Predictions under alternative behavioral forces}

Besides expected payoff maximization, other behavioral motives may play a role as well. This alters the comparative statics in interest alignment that one could expect. First consider the delegation case in isolation. Restricted delegation may perform less well than predicted, because the worker may purposely make a suboptimal project decision if the manager intentionally decided to restrict his choice set to $\Delta=\{A, B\}$. Such hidden costs of control have been observed in related principalagent settings with no private information (cf. Falk and Kosfeld, 2006). Punishment may in principle occur in states 1 through 3 (in state 4 a deviation from $B$, i.e. the worker's best project 
in $\Delta=\{A, B\}$, to $A$ would be rewarding the manager). Arguably the most salient state for the worker to do so is $t=3$; in this state the principal's restriction to $\Delta=\{A, B\}$ really hurts both. If the worker only punishes in this state by suboptimally choosing $A$ instead of $B$, the principal prefers full delegation whenever $q>\frac{21}{31} \approx 0.68$. Given our parameter choices, this drop (from $\frac{7}{10}$ to $\left.\frac{21}{31}\right)$ in the cutoff between full delegation and restricted delegation is likely to have little effect. ${ }^{9}$

The other side of the same coin is that the worker may reward the principal for not restricting the choice set to $\Delta=\{A, B\}$ when it is theoretically optimal to do so. Full delegation signals trust and can be considered a kind act, to which a reciprocal agent might react with using her authority more "responsibly". In particular, the worker may choose project $B$ as a compromise in state 4 if authority is fully delegated, to reward the manager for her trust. If the worker indeed does so, the principal never prefers $\Delta=\{A, B\}$. If only a fraction $r \in[0,1]$ of workers behaves reciprocal in this way, the cutoff between full and restricted delegation becomes $\frac{7-7 r}{10-7 r}$. For $r \geq \frac{5}{14} \simeq 0.36$ we would then also expect to observe full delegation in the low interest alignment case $q=\frac{3}{5} \cdot{ }^{10}$ Given that this requires a sizable fraction of sufficiently reciprocal types, we a priori do not expect that in our setting hidden costs of control (or the flip side, hidden benefits of autonomy) overturn the earlier comparative statics predictions.

A behavioral force that may affect the communication equilibria is lying aversion. When sending their message workers may be averse to lying, especially when the costs to the manager are substantial relative to the benefits of the worker if the lie is believed (cf. Gneezy, 2005). Lying aversion in particular has a bite when $t=4$ and a worker acting strategically has an incentive to let the manager believe that $t=3$. Suppose a fraction $\lambda$ of workers is sufficiently lying averse and always speaks the truth, thus also when $t=4$. This lowers the requirement on $q$ for Com-(3) to be possible: $q>\frac{33(1-\lambda)}{10+33(1-\lambda)}$ is then needed to secure that after message $m_{3}$ the boss is willing to

\footnotetext{
${ }^{9} \mathrm{~A}$ behavioral force that may make restricted delegation relatively more attractive as compared to full delegation is anticipated regret. Regret may operate in two ways. The manager may regret having restricted the worker (by excluding project $C$ ) when it turns out ex post that the state equals $t=3$. Likewise, the manager may regret having given full discretion to the worker when it turns out ex post that $t=4$. The tradeoff between these two types of anticipated regret depends on the relative weight attached to them, the monetary payoffs in the various states and the likelihood of each state occuring. The auction experiment of Filiz-Ozbay and Ozbay (2007) suggests that regretting too much discretion gets a relative larger weight; they find that subjects feel regret from not being aggressive enough ('loser regret' of bidding too low) but not from being too aggressive ('winner regret' of bidding too high). Our parameter choices make regretting full delegation also more salient. Fehr et al. (2013) indeed find that regret aversion, exhibited through a distaste for being overruled, provides a behavioral explanation for under-delegation in their setup (in our setup this would correspond to either restricted delegation or communication).

${ }^{10}$ In Oosterbeek et al (2011) a related argument is formally developed using intention based reciprocity in a multitasking context. Translated to the current setup, the argument is that the smaller $q$, the stronger full delegation is a signal of trust, thus warranting a stronger reciprocal response. Therefore, behaviorally one may expect that full delegation does not increase with $q$.
} 
choose $C$. For $q=\frac{3}{4}$ then at least a fraction of $\lambda=\frac{1}{11}$ lying averse people are required, for $q=\frac{3}{5}$ this becomes $\lambda=\frac{6}{11}$. Given earlier lying experiments, the former requirement is rather likely to be satisfied, the latter is not. Note that in that case also for $q=\frac{3}{4}$ optimal delegation and optimal communication coincide. ${ }^{11}$

Similarly, when there is a sufficient fraction of managers that is credulous and always believes the message received and act accordingly, the Com-(3) message strategy of the worker can be sustained for lower values of $q \leq \frac{33}{43}$. In that case a rational, non-credulous manager always chooses A after $m_{3}$. For the worker to still send this message when $t=4$, it is then required that the fraction of credulous managers exceeds $\frac{7}{11}$. This a priori seems too stringent for credulity to have an impact. ${ }^{12}$

Overall, lying aversion seems to be the most relevant alternative behavioral force that may overturn comparative statics. A priori one would then expect that the amount of information transmission under communication decreases when interest alignment drops from strong $\left(q=\frac{3}{4}\right)$ to weak $\left(q=\frac{3}{5}\right)$. Moreover, with lying aversion the comparative advantage of delegation over communication vanishes for both values of $q$.

\section{Experimental design}

The purpose of our experiment is to test the comparative statics of centralization and delegation with the level of interest alignment. We therefore considered two different values of $q$ : under weak interest alignment $q=\frac{3}{5}=0.6$, while under strong interest alignment $q=\frac{3}{4}=0.75$. Moreover, before subjects actually had to choose between centralization and delegation, we wanted them to have some experience already with these two institutions in isolation. To account for potential order effects, we used the order of these 'exogenous institution' games as second treatment dimension. Table 2 provides an overview of our 2 by 2 treatments design.

The experiment was conducted at the University of Amsterdam and was programmed using the z-tree programming package by Fischbacher (2007). In total 230 subjects participated. For each treatment we ran 2 or 3 sessions, where the number of participants per session ranged from 20 to 30. As the sessions differed in the number of participants, in each session we had either two or three matching groups of either 8,10 , or 12 subjects. Subjects were only matched within their matching

\footnotetext{
${ }^{11}$ Like lying aversion, level- $k$ analysis may also rationalize over-communication and, thereby, under-delegation; see Lai and Lim (2012) for an elaborate discussion of level- $k$ in the context of their model.

${ }^{12}$ For the standard Crawford and Sobel framework, Ottaviani (2000) shows that there is a fully revealing equilibrium as soon as there is a positive fraction of credulous (or 'naive' in his wording) managers.
} 
groups, so matching group averages can be taken as strictly independent observations. Table 2 also lists the number of matching groups and thus independent observations per treatment.

Each session consisted of four incentivized parts and an ex post questionnaire. After reading the instructions for part 1 and completing some control questions, each subject learned her/his role: either Manager or Worker. Subjects kept the same role throughout the experiment. The experiment was framed in an organizational setting (see Appendix B for sample instructions). Overall earnings equalled the sum of earnings in parts I through IV. The conversion rate was 250 points for 1 euro.

Table 2: Treatment variations

\begin{tabular}{cccccccc}
\hline \hline$q$ & Treatment & \# subj. & \# groups & Part I & Part II & Part III & Part IV \\
\hline 0.6 & CD60 & 60 & 6 & C & D & C vs. D & lying aversion \\
0.6 & DC60 & 50 & 6 & D & C & C vs. D & lying aversion \\
0.75 & CD75 & 66 & 7 & C & D & C vs. D & lying aversion \\
0.75 & DC75 & 54 & 6 & D & C & C vs. D & lying aversion \\
\hline \hline
\end{tabular}

Parts I and II of the experiment gave subjects the opportunity to gain experience with Centralization and Delegation in isolation. In the Centralization game the subject in the role of manager took the project decision, but before doing so the subject in the role of worker sent a message about the actual state of the world (of which only the worker was informed). We restricted the worker to use exactly one of the following eight messages: "The state is $t$ " for $t=1,2,3,4$, "I recommend project $P$ " for $P=A, B, C$ and "I make no recommendation". Subjects were explicitly informed in the instructions that: "The set of available messages does not depend on the actual state; so irrespective of the actual state, the worker can always choose one of the above eight messages." Our choice for this particular set of messages was partly inspired by Sobel (2013). He notes that linguists typically make a distinction between the referential function of communication ('reporting the facts') and the conative function of communication ('giving advice'). Another reason was to explore whether subjects shy away from explicitly telling a lie in the conflict state $t=4$, e.g. by then using "I recommend project $C$ ". ${ }^{13}$

\footnotetext{
${ }^{13}$ Serra-Garcia, Van Damme, and Potters (2011) compare a setting with precise communication about the state of the world to a setting with vague communication using a public good game with three values: low, intermediate or high. Under precise communication subjects can send "The value is $v^{\text {" for }} v=$ low, intermediate, high. Under vague communication subjects are also allowed to send for example the message "The value is intermediate or high". In the vague treatment subjects can refrain from lying by sending a vague message. They find that when vague communication is allowed the leader turns to vague messages in the conflict state.
} 
In the Delegation game the worker took the project decision, but before doing so the manager could restrict the worker's choice options. In part III of each session the manager first had to choose between Centralization and Delegation and then the corresponding subgame was played.

Each of the parts I, II and III consisted of 20 periods. In each period the manager and the worker were anonymously and randomly rematched within their matching group. At the end of each period, a summary of the manager's and worker's decisions and the resulting payoffs in that period was shown to them. These payoffs equalled the number of points of the implemented project given the true state of the world as reflected in Table 1. Subjects did not receive information on the behavior of other managers and workers. Furthermore, before making any decision in part III, subjects received an overview of the decisions made in the first two parts of the experiment. The overall payoffs of each subject in each part was equal to the sum of points earned in all 20 periods.

We included a part IV in the experiment with the aim of measuring lying aversion of workers as well as credulity of managers in an incentivized way. We used an adjusted version of the experiment by Gneezy et al (2013). The exact setup is discussed in Section 5 where we discuss our findings. We ended the experiment with an ex post questionnaire. Besides background characteristics, we included Likert type statements to measure preferences for control, power, authority and reciprocity. Most importantly, to complement the incentivized measure of part IV we also included the 10 items from Lundquist et al (2009) to measure attitudes towards lying.

Sessions lasted around two hours. Average earnings equalled 20 euros, with a minimum of 13.8 euros and a maximum of 28.3 euros.

\section{Results}

This section presents an overview of the experimental results. First we look at the choices made in parts I and II where delegation and centralization are studied in isolation. Subsequently we will consider subjects' behavior when the manager has the opportunity to choose between centralization and delegation (part III data). Unless indicated otherwise, all tables and test statistics are based on matching group averages. Moreover, we always pool the data of the two different orders given that the two orders are balanced over the two different values of $q$. 


\subsection{Delegation}

In this subsection we consider the results under delegation when exogenously imposed. The manager and the worker interact for 20 periods. To account for learning effects we focus on the decisions taken in the last 10 periods. Following the theoretical predictions, we distinguish three types of outcomes based on the number of projects that are allowed: Del-(3), Del-(2) and Del-(1). Table 3 summarizes the results. Managers restrict the choice set of workers more frequently under weak alignment than under strong alignment. In the latter case managers choose to delegate full decision power to workers in $66 \%$ of the cases, while under weak alignment this only happens in $13 \%$ of the cases. ${ }^{14}$ The difference is highly significant according to a ranksum test $(p<0.001)$. Another way to illustrate the exact same finding is to look at the average number of allowed projects. This equals 2.6 under strong interest alignment, significantly higher than the 1.9 observed under weak alignment $(p<0.001)$. These comparative statics findings are in line with theoretical predictions.

Table 3: Delegation decision manager

\begin{tabular}{lccc}
\hline \hline & $q=0.75$ & $q=0.6$ & $p$-value \\
\hline Del-(3) & $66 \%$ & $13 \%$ & $<0.001$ \\
Del-(2) & $29 \%$ & $68 \%$ & $<0.001$ \\
Del-(1) & $5 \%$ & $19 \%$ & 0.03 \\
& & & \\
$\#$ of allowed projects & 2.6 & 1.9 & $<0.001$ \\
\hline \hline & Note: The $p$-values in the last column are from ranksum tests performed at \\
matching group averages. For the top part the three tests are not independent.
\end{tabular}

As to the worker's project choice (unreported), in the far majority of cases the worker chooses the project that maximizes his payoffs given the state and his allowed choice set. In the conflict state $t=4$, the worker chooses project $\mathrm{C}$ under full delegation in about $75-85 \%$ of the cases, even though this project really hurts the manager in this situation. In the remaining $15-25 \%$ of the cases the worker chooses the compromise project B. The worker is more likely to do so under weak interest alignment. As discussed in Section 2, a potential reason might be that full delegation is a stronger signal of trust the lower $q$ is, leading to a stronger reciprocal reaction. If restricted to either project $\mathrm{A}$ or $\mathrm{B}$ in state 3 , the worker chooses $\mathrm{A}$ in only $5-7 \%$ of the cases. We thus do not

\footnotetext{
${ }^{14}$ In almost all of the Del-(2) cases managers restrict the choice set to projects A and B. In almost all of the Del-(1) cases this is either project $\mathrm{A}$ or project $\mathrm{B}$.
} 
find large hidden costs of control in our setting. By and large, under delegation managers and workers behave reasonably well in line with standard theoretical predictions.

We next compare actual payoffs with predicted payoffs. Under strong alignment theory predicts full delegation and expected payoffs for the manager of $10+\frac{250}{3} q=72.5$ per period. For the worker this is $120-\frac{80}{3} q=100$. Under weak interest alignment Del-(2) is predicted with expected payoffs of $80-\frac{50}{3} q=70$ per period for both the manager and the worker. Table 4 depicts the actual and predicted average payoffs over the final ten periods; here the predicted values differ slightly from the ones just discussed as these are calculated based on the actual realized draws of states (rather than their theoretical distribution as reflected by $q$ ). The table reveals that actual payoffs fall short of predicted payoffs. Except for managers under strong interest alignment, these differences are significant. Under delegation subjects thus earn less than theory predicts.

Table 4: Actual and predicted payoffs under delegation

\begin{tabular}{lccc}
\hline \hline & $q=0.75$ & $q=0.6$ & $p$-value \\
\hline Manager's payoffs & & & \\
Actual & 71.3 & 64.0 & 0.001 \\
Predicted & 72.3 & 68.1 & $<0.001$ \\
Act. vs Pred. ( $p$-value) & 0.80 & 0.00 & \\
& & & \\
Worker's payoffs & & & $<0.001$ \\
Actual & 86.9 & 62.4 & $<0.001$ \\
Predicted & 100.1 & 68.1 & \\
Act. vs Pred. $(p$-value $)$ & 0.00 & 0.03 & \\
\hline \hline
\end{tabular}

Note: The $p$-values in the last column are from ranksum tests using matching group averages. The other $p$-values are from signed rank tests.

\subsection{Centralization}

We next turn to the results under centralization, again focusing on the decisions taken in the last 10 periods (out of 20). Table 5 provides an overview of the messages sent by workers. (The table also reports best responses in the columns labelled BR; these are discussed later. The corresponding rows " "1" equivalent to "A" ' et cetera, are then explained as well.) 
Table 5: Worker's actual messages and best responses by treatment and state

\begin{tabular}{|c|c|c|c|c|c|c|c|c|c|}
\hline \multirow{2}{*}{\multicolumn{2}{|c|}{ Strong alignment $(q=0.75)$}} & \multicolumn{2}{|c|}{ State 1} & \multicolumn{2}{|c|}{ State 2} & \multicolumn{2}{|c|}{ State 3} & \multicolumn{2}{|c|}{ State 4} \\
\hline & & Actual & $\mathrm{BR}$ & Actual & $\mathrm{BR}$ & Actual & $\mathrm{BR}$ & Actual & $\mathrm{BR}$ \\
\hline \multirow[t]{3}{*}{ M1 } & The state is 1 & 84 & $38[38]$ & 0 & $0[0]$ & 0 & $0[0]$ & 0 & $0[0]$ \\
\hline & I recommend project $\mathrm{A}$ & 14 & $8[2]$ & 0 & $0[0]$ & 1 & $0[0]$ & 1 & $0[0]$ \\
\hline & "1" equivalent to "A" & & $54[52]$ & & $0[0]$ & & $0[0]$ & & $0[0]$ \\
\hline \multirow[t]{3}{*}{ M2 } & The state is 2 & 0 & $0[0]$ & 84 & $54[47]$ & 2 & $0[0]$ & 30 & $31[13]$ \\
\hline & I recommend project $\mathrm{B}$ & 2 & $0[0]$ & 16 & $0[0]$ & 0 & $0[0]$ & 13 & $0[0]$ \\
\hline & "2" equivalent to "B" & & $0[0]$ & & $46[46]$ & & $0[0]$ & & $23[12]$ \\
\hline \multirow[t]{2}{*}{ M3 } & The state is 3 & 0 & $0[0]$ & 0 & $0[0]$ & 74 & $92[69]$ & 45 & $38[17]$ \\
\hline & I recommend project $\mathrm{C}$ & 0 & $0[0]$ & 0 & $0[0]$ & 19 & $8[2]$ & 9 & $8[0]$ \\
\hline \multirow[t]{2}{*}{ M4 } & The state is 4 & 0 & n.a. & 1 & n.a. & 1 & n.a. & 1 & n.a. \\
\hline & I make no recommendation & 0 & n.a. & 0 & n.a. & 3 & n.a. & 2 & n.a. \\
\hline \multicolumn{10}{|c|}{ Weak alignment $(\mathrm{q}=0.6)$} \\
\hline \multirow[t]{3}{*}{ M1 } & The state is 1 & 77 & $42[35]$ & 0 & $0[0]$ & 0 & $0[0]$ & $0[0]$ & $0[0]$ \\
\hline & I recommend project $\mathrm{A}$ & 18 & $0[0]$ & 0 & $0[0]$ & 0 & $0[0]$ & $1[0]$ & $0[0]$ \\
\hline & "1" equivalent to "A" & & $58[55]$ & & $0[0]$ & & $0[0]$ & & $0[0]$ \\
\hline \multirow[t]{3}{*}{ M2 } & The state is 2 & 0 & $0[0]$ & 82 & $67[58]$ & 5 & $0[0]$ & 50 & $33[14]$ \\
\hline & I recommend project $\mathrm{B}$ & 0 & $0[0]$ & 17 & $8[2]$ & 1 & $0[0]$ & 16 & $8[0]$ \\
\hline & "2" equivalent to "B" & & $0[0]$ & & $17[16]$ & & $0[0]$ & & $8[6]$ \\
\hline \multirow[t]{2}{*}{ M3 } & The state is 3 & 0 & $0[0]$ & 0 & $0[0]$ & 76 & $75[58]$ & 21 & $33[5]$ \\
\hline & I recommend project $\mathrm{C}$ & 2 & $0[0]$ & 0 & 8 & 16 & $25[3]$ & 7 & $17[1]$ \\
\hline \multirow[t]{2}{*}{ M4 } & The state is 4 & 0 & n.a. & 0 & n.a. & 1 & n.a. & 2 & n.a. \\
\hline & I make no recommendation & 3 & n.a. & 1 & n.a. & 2 & n.a. & 2 & n.a. \\
\hline
\end{tabular}

Note: Percentages in table are based on matching group averages. n.a. means that best responses are not available because of insufficient actual occurrences of messages M4. Numbers within brackets reflect the best responses that correspond to the actual message sent.

Workers can either send a message about the state or recommend a project. Workers can also choose not to make a recommendation. Overall in $81 \%$ of the cases under strong interest alignment 
workers send a message about the state. For weak interest alignment this is $76 \%$. In the three congruent states 1, 2 and 3, workers almost always announce the true state or recommend the project that is best for both. However, in state 4 where interests conflict, workers explicitly lie in the majority of cases ( $75 \%$ resp. $71 \%$ under strong resp. weak alignment), by stating that the state equals either 2 or 3 . Only in $4 \%$ of the $q=0.75$ cases ( $5 \%$ for $q=0.6$ ) the worker can be considered truly honest, by sending one of the messages "the state is 4", "I recommend project A", or "I make no recommendation". Besides being truly honest, in the conflict state subjects may shy away from explicitly telling a lie by making a recommendation (for B or C) instead of sending a message about the state. For state 4 the percentage of messages that reports about the state equals $76 \%$ (73\%) under strong (weak) alignment, while in the three congruent states this is $81 \%$ (80\%). Hence, in the conflict state subjects are slightly less inclined to make a factual statement about the state. But according to signrank tests differences are marginally significant only under weak interest alignment ( $\mathrm{p}$-value of 0.51 (0.09) under strong (weak) alignment). Lying aversion therefore does not seem to play a major role.

Given the pattern in Table 5, for ease of reference we sometimes bundle the different types of messages in the four classes M1 through M4. M1 then corresponds to "The state is 1" and "I recommend project $\mathrm{A}^{\prime}$, et cetera (see the first column in Table 5). 
Table 6: Manager's response to received messages under centralization by treatment

\begin{tabular}{|c|c|c|c|c|c|c|c|}
\hline & & \multicolumn{2}{|c|}{ Project A } & \multicolumn{2}{|c|}{ Project B } & \multicolumn{2}{|c|}{ Project $\mathrm{C}$} \\
\hline \multicolumn{2}{|c|}{ Strong alignment $(\mathrm{q}=0.75)$} & Actual & $\mathrm{BR}$ & Actual & $\mathrm{BR}$ & Actual & $\mathrm{BR}$ \\
\hline \multirow[t]{2}{*}{ M1 } & The state is 1 & 99 & $100[99]$ & 0 & $0[0]$ & 1 & $0[0]$ \\
\hline & I recommend project $\mathrm{A}$ & 100 & $100[100]$ & 0 & $0[0]$ & 0 & $0[0]$ \\
\hline \multirow[t]{2}{*}{ M2 } & The state is 2 & 0 & $0[0]$ & 100 & $100[100]$ & 0 & $0[0]$ \\
\hline & I recommend project $\mathrm{B}$ & 9 & $30[3]$ & 91 & $70[64]$ & 0 & $0[0]$ \\
\hline \multirow[t]{2}{*}{ M3 } & The state is 3 & 39 & $0[0]$ & 7 & $0[0]$ & 54 & $100[54]$ \\
\hline & I recommend project $\mathrm{C}$ & 63 & $17[13]$ & 15 & $0[0]$ & 22 & $83[20]$ \\
\hline \multirow[t]{2}{*}{ M4 } & The state is 4 & 100 & n.a. & 0 & n.a. & 0 & n.a. \\
\hline & I make no recommendation & 10 & n.a. & 83 & n.a. & 7 & n.a. \\
\hline \multicolumn{8}{|c|}{ Weak alignment $(q=0.6)$} \\
\hline \multirow[t]{2}{*}{ M1 } & The state is 1 & 100 & $100[100]$ & 0 & 0 & 0 & $0[0]$ \\
\hline & I recommend project $\mathrm{A}$ & 98 & $100[98]$ & 2 & $0[0]$ & 0 & $0[0]$ \\
\hline \multirow[t]{2}{*}{ M2 } & The state is 2 & 5 & $0[0]$ & 95 & $100[95]$ & 0 & $0[0]$ \\
\hline & I recommend project B & 22 & $45[13]$ & 78 & $55[46]$ & 0 & $0[0]$ \\
\hline \multirow[t]{2}{*}{ M3 } & The state is 3 & 44 & $25[13]$ & 10 & $0[0]$ & 45 & $75[36]$ \\
\hline & I recommend project $\mathrm{C}$ & 62 & $73[43]$ & 16 & $0[0]$ & 22 & $27[0]$ \\
\hline \multirow[t]{2}{*}{ M4 } & The state is 4 & 88 & n.a. & 13 & n.a. & 0 & n.a. \\
\hline & I make no recommendation & 39 & n.a. & 48 & n.a. & 13 & n.a. \\
\hline
\end{tabular}

Note: Percentages in table are based on matching group averages. n.a. means that best responses are not vailable because of insufficient actual occurrences of messages M4. Numbers within brackets reflect the best responses that correspond to the actual project implemented.

Next we look at how the manager reacts to the worker's information. Table 6 shows for each treatment separately, the manager's project choice (in the columns) given the worker's message (in rows). Again the columns with best responses BR are discussed later. Messages that the state equals 1 or that project $\mathrm{A}$ is recommended, almost always lead to a choice for $\mathrm{A}$. An announcement that the state equals 2 leads to project B being implemented almost for sure. Yet a recommendation for this project is treated slightly more skeptical, leading to a choice for project A now and then. 
Likewise, a recommendation for project $\mathrm{C}$ is treated more skeptical than a message that the state equals 3 is. After both messages project $\mathrm{A}$ is chosen frequently (besides project $\mathrm{C}$ ), but this happens much more often after the recommendation than after the factual statement.

Previously we have seen that (under weak alignment) in the conflict state workers tend to send less often a message about the state than in the congruent states. In line with this, the above observations suggest that managers respond somewhat differently to a message about the state than to a message containing a recommendation. To test this more carefully, we define "following the worker's message" as taking the message to be truthful or as sincere advice, and determine the ratio of "following the worker's message about state $t$ " and "following the worker's message recommending project $P^{\prime \prime}$. We do so for the three relevant cases $\mathrm{M} 1(t=1, P=A), \mathrm{M} 2(t=2$, $P=B)$, and M3 $(t=3, P=C)$. The ratio under strong (weak) alignment equals 0.99 (1.02) for M1, 1.13 (1.28) for M2, and 1.71 (1.54) for M3. According to a Cuzick test for trends the positive trend in the ratio is only significant for the strong alignment treatment ( $\mathrm{p}$-value 0.004 under strong alignment and 0.614 under weak alignment). We thus find some weak evidence that factual statements are treated differently than recommendations.

Taken together, and ignoring the minor differences between factual statements and recommendations, the manager's behavior can be roughly summarized as follows. After M1 the manager always follows and implements project A. After M2 the manager is very likely to follow by choosing project B; only now and then she opts for project A. But if M3 is received, the manager's behavior is more mixed. In that case she is about equally likely to follow by choosing $\mathrm{C}$, as not to follow and opting for project A. ${ }^{15}$

The (pure strategy) equilibrium predictions for the two treatments are the same: the worker's message strategy is such that the manager can only separate state $\{1\}$ from the states $\{2,3,4\}$ (which he cannot further distinguish). In the former case he chooses project $\mathrm{A}$, in the latter case project B. However, this outcome is not fully reflected in the actual data. On the one hand, the worker's message contains more information than theory predicts. In particular, the worker almost always chooses different messages in states 2 and 3, viz. M2 and M3 respectively (cf. Table 5). Therefore, when M2 is observed, the manager can infer that the state equals either 2 or 4 , but (almost surely) not 3. Similarly so, if M3 is received, the manager knows that the state is either 3

\footnotetext{
${ }^{15}$ Messages in the M4 category are hardly ever received (see Table 5), making reliable inferences about the manager's reaction difficult. Moreover, it is a priori not clear what following the worker's message "I make no recommendation" would entail. If anything, note that the manager almost always follows the factual statement that "the state is 4 " by choosing project $\mathrm{A}$.
} 
or 4, but not 2. Both M2 and M3 thus contain more information about the true state than theory predicts. On the other hand, the manager is also more responsive to the messages than theory would predict. According to theory, the manager should implement project B independent of whether he observes M2 or M3. The manager indeed does so after M2, but only very infrequently after M3. She then typically mixes between projects A and C. A potential explanation for this behavior might be the more informative nature of the worker's messages. Being more responsive might be a best response to messages being more informative. Yet another explanation might be that managers are credulous.

For a better understanding of what is driving the worker's and manager's behavior, we next consider their best responses. To determine the worker's best response, we take managers' average behavior in a matching group as the manager's strategy (as before we focus on the final 10 periods). For each state of the world we subsequently determine which message would maximize the worker's payoffs and label this as the best response message. In a number of instances (i.e. matching groups) two different messages induce the manager to make the same choice. For instance, after both "The state is 1 " and "I recommend project A" the manager chooses project $\mathrm{A}$. In that case there is no unique best response and we label that as: "1" is equivalent to "A". ${ }^{16}$ Both messages in category M1 are then in line with best response. The columns labelled BR in the earlier Table 5 report the average (of the matching group averages) percentage of cases in which the message in question would be the worker's best response. The fraction of actual messages that is in line with the best response is reflected in brackets in the columns labelled BR. We leave the messages from the M4 category out of the analysis, as these are hardly ever sent so no reliable estimates of the manager's response to these messages can be inferred.

In state 1 the worker is almost always best responding. The worker then should either send "the state is 1 " or "I recommend project A", and often both are equally good (in overall $54 \%$ of the cases that state 1 occurs). Indeed, the worker hardly ever sends a message outside M1. Similar observations can be made regarding states 2 and 3, where workers should and indeed do pick a message from M2 and M3, respectively. However, while under strong alignment in state 3 workers

\footnotetext{
${ }^{16}$ In some cases "I recommend project C" also leads to the manager choosing project A. Again there is no unique best response in these cases. For ease of presentation we do not label these cases separately, but include them either in category "The state is 1 " or in category " 1 " is equivalent to "A". More precisely, under strong (weak) alignment in category "The state is 1 " the fraction $38 \%$ (42\%) is a combined fraction; $8 \%(25 \%)$ have "The state is 1 " as unique best response, while $31 \%(17 \%)$ "The state is 1 " and "I recommend project C" are best responses. Furthermore, under weak alignment in category " 1 " is equivalent to "A" $42 \%$ have "The state is 1 " and "I recommend project A" as best response, while for $17 \%$ "The state is 1 ", "I recommend project A", and "I recommend project C" are best responses.
} 
rightfully choose a message from M3, they tend to send more often a recommendation and less often a message about the state than would be optimal given the manager's behavior. Workers deviate from best response when the state is 4 . Then, under strong interest alignment, workers actually send M3 somewhat more often and M2 somewhat less often than would be optimal given managers' behavior. In the weak alignment treatment we see the opposite. There M2 is sent more often and M3 less often as compared to best response. In both treatments workers send a message recommending project B more often than would be optimal.

In a similar way we determine the manager's best response. Now we take workers' average behavior in a matching group as the worker's strategy. We use this to determine for each message received which project maximizes the manager's payoff. From Table 6 it can be observed that the manager best responds to messages in M1. This is also the case when message "the state is 2" is received. However, after "I recommend project B" the manager is too credulous. Under both strong and weak interest alignment project B is chosen too often; the manager would benefit from choosing project A more frequently. In contrast, the factual statement that the state equals 3 is on average treated too skeptical. Managers then frequently opt for project A while project $\mathrm{C}$ is either always (under strong alignment) or very often (under weak alignment) the best response. A similar observation holds when the worker "recommends project $\mathrm{C}$ " under strong interest alignment; this advice is treated too skeptical. Under weak alignment such an advice is by and large interpreted appropriately.

Overall the following picture emerges from the best response analysis. The worker is rightfully more informative than in the Com-(2) equilibrium, by using different messages M1, M2 and M3 in states 1, 2 and 3 respectively. In state 4 the worker chooses between M2 and M3, thus not sending M1. Given the manager's response this is indeed the right thing to do. The exact frequencies with which M2 and M3 are then chosen do not match the best responses, but deviations are in opposite directions for the two interest alignment treatments. In that sense the worker does not seem to make a systematic mistake towards one or the other, but rather miscalibrates in the exact mixing probabilities. The stronger responsiveness of managers is by and large a result of messages being more informative. We do not find systematic evidence that managers are too credulous. We return to this in Section 5.

We end this subsection with comparing the actual payoffs to the predicted payoffs. In both treatments Com-(2) is predicted with expected payoffs of $80-\frac{50}{3} q$ per period for manager and worker alike. They are thus expected to receive 67.5 on average when $q=\frac{3}{4}$ and 70 in case $\frac{3}{5}$. 
Table 7 depicts the actual and predicted payoffs; again the predicted values differ slightly from the ones above due to the actual realized draws of states. Except for workers under weak interest alignment (where we find no significant differences with predicted payoffs), the table shows that both the manager and the worker obtain significantly larger payoffs than theory predicts. Overall, under centralization subjects thus earn (weakly) more than predicted. (The table also reports the predicted payoffs under a mixed strategy equilibrium; these are discussed in Section 5.)

Table 7: Actual and predicted payoffs under centralization

\begin{tabular}{lccc}
\hline \hline & $q=0.75$ & $q=0.6$ & $p$-value \\
\hline Manager's payoffs & 72.0 & 74.4 & 0.34 \\
Actual & 67.3 & 69.0 & $<0.001$ \\
Predicted & 74.3 & 74.8 & $<0.001$ \\
Predicted Mixed & 0.03 & 0.02 & \\
Actual vs Predicted ( $p$-value) & 0.17 & 0.81 & \\
Actual vs Predicted Mixed $(p$-value) & & & \\
& & & \\
Worker's payoffs & & & \\
Actual & 74.7 & 68.4 & 0.09 \\
Predicted & 67.3 & 69.0 & $<0.001$ \\
Predicted Mixed & 81.0 & 80.3 & $<0.001$ \\
Actual vs Predicted ( $p$-value) & 0.01 & 0.81 & \\
Actual vs Predicted Mixed $(p$-value) & 0.02 & 0.00 & \\
\hline \hline
\end{tabular}

Note: The $p$-values in the last column are from ranksum tests using matching group averages. The other $p$-values are from signed rank tests.

\subsection{Choice between centralization and delegation}

In the third part of the experiment, the manager chooses whether to delegate the project choice to the worker or to make the choice himself. According to standard theory, the manager should always delegate under strong interest alignment while under weak interest alignment he would be indifferent. A priori one would thus expect more delegation when interests are stronger aligned.

The data reveal that over all 20 periods, the manager delegates in $40 \%$ of the cases under 
strong interest alignment and in $36 \%$ under weak interest alignment. The difference between treatments is insignificant according to a ranksum test $(p=0.48)$. Moreover, the delegation fraction is significantly lower than $50 \%$ (under weak alignment $p=0.01$ according to a signrank test, while $p=0.06$ under strong alignment), implying that the manager chooses less often to delegate than to communicate. We therefore do not find support for the theoretical prediction that delegation is more likely especially under strong interest alignment. In the previous subsections we have seen that actual payoffs under Delegation are typically smaller than predicted, while under Centralization this is the other way around. This may explain the low proportion of delegation. Indeed, if we look at the correlation between delegation fractions and the relative profits of delegation vs. communication in the first two parts, we find that managers who in previous parts received a higher profit under delegation than under centralization are more likely to delegate in part III. Under strong interest alignment the (Spearman) correlation equals 0.48 whereas under weak interest alignment it equals 0.54. In both cases it is highly significant $(p<0.001) .{ }^{17}$

Part III behavior under each of the two organizational designs is closely in line with subjects' behavior when the design at hand is exogenously given (as in parts I and II). The endogenous choice between delegation and centralization thus appears to have little impact on behavior per se. In case delegation is chosen, the manager puts restrictions on the worker similar as in Table 2. The average number of allowed projects equals 2.8 under strong alignment, significantly higher than the 2.0 under weak alignment $(p<0.001) .{ }^{18}$ The worker again typically chooses the project that maximizes his payoffs given his allowed choice set and the state.

Under communication the worker almost always sends message $\mathrm{M} t$ in the congruent states $t \in\{1,2,3\}$. In the conflict state 4, the worker mixes between M2 and M3. Under strong alignment each of these two messages is then chosen about equally often, under weak alignment M2 is chosen substantially more often than M3 is. The manager almost always follows M1 by implementing project A, and in the far majority of cases implements project B after M2. Finally, if M3 is received, then the manager implements Project $\mathrm{C}$ in about $50 \%$ of the cases independent of the level of interest alignment. Overall the worker is thus equally willing to share information as in the exogenous communication part, even though now delegation was an explicit option but forfeited by the manager.

\footnotetext{
${ }^{17}$ No significant correlations are found with items in the ex post questionnaire that relate to control, power and authority.

${ }^{18}$ The distributions over Del-(3), Del-(2) and Del-(1) are now somewhat more pronounced: $82 \%, 16 \%$ and $2 \%$ for $q=\frac{3}{4}$ and $8 \%, 83 \%$ and $9 \%$ for $q=\frac{3}{5}$.
} 
Average payoffs also follow the pattern observed in the exogenous parts. As Table 8 reveals, the manager earns about the same under centralization as under delegation. Hence, the manager should be indifferent between centralization and delegation. The worker receives a significantly larger average payoff under delegation than under centralization in the strong alignment treatment. In case of weak alignment differences are not significant.

Table 8: Actual payoffs by organizational design

\begin{tabular}{lccc}
\hline \hline & $q=0.75$ & $q=0.6$ & $p$-value \\
\hline Manager's payoffs & & & \\
Delegation & 74.2 & 65.4 & 0.09 \\
Centralization & 76.5 & 71.6 & 0.33 \\
Del. vs Cen. ( $p$-value) & 0.17 & 0.39 & \\
& & & \\
Worker's payoffs & & & \\
Delegation & 91.5 & 69.0 & 0.00 \\
Centralization & 75.4 & 67.8 & 0.10 \\
Del. vs Cen. ( $p$-value) & 0.00 & 0.75 & \\
\hline \hline
\end{tabular}

Note: The $p$-values in the last column are from ranksum tests using matching group averages. The other $p$-values are from signed rank tests.

\section{Discussion}

Our experimental findings reveal that workers send more informative messages under communication than predicted. In this section we explore potential explanations for this finding.

\subsection{Lying aversion and credulity}

\subsubsection{Lying aversion}

A first potential explanation for the finding that communication is more informative than theory predicts, is that workers are lying averse and therefore less inclined to lie about the true state of the world. The results reported in Table 5, however, make this an unlikely explanation. There we already observed that in the conflict state 4 workers lie in about $95 \%$ of the cases (by saying something different than "The state is 4", "I recommend project A", or "I make no recommenda- 
tion"). In the congruent states where interests are aligned, the workers lie in only $1-2 \%$ of the cases. The overall pattern is thus very much in line with standard strategic motives. This is somewhat surprising. As Sobel (forthcoming) notes, the existing experimental evidence namely suggests that agents are more honest and more credulous than equilibrium theory suggests, with lying aversion being one of the main explanations. It thus begs the question whether either our subject pool is somewhat special, or rather the strategic situation that we consider. The latter may be due to the fact that in our setting sender and receiver often have common interests and the sender is best off telling the truth (i.e. in the congruent states). This may make lying in the conflict state less problematic, as this state only occurs now and then. This is different in experimental tests of the standard Crawford-Sobel framework like Cai and Wang (2006), where the alignment of interests is state independent and the sender effectively always has an incentive to portray the state differently that it actually is. Another factor here might be that our design is based on messages of a fixed format. Typically this leads to more lying than a free format chat does (cf. Charness and Dufwenberg, 2010).

Part IV of our experiment sheds some light on whether our subject pool is special. Here we measure lying aversion of workers (and credulity of managers, see the next subsection) using an adjusted version of the experiment by Gneezy et al (2013). In particular, workers in the earlier parts are assigned role A, while former managers are assigned role B. Each randomly matched pair of $\mathrm{A}$ and $\mathrm{B}$ is assigned an integer out of 1 to 6 , of which only subject $\mathrm{A}$ is informed. Subject $\mathrm{A}$ sends a message $m$ about the assigned number to subject $\mathrm{B}$. The higher the message she sends, the more A earns, irrespective of the actual number assigned to the pair (in particular, A earns $500+100 \mathrm{~m}$ ). Subject B can either follow ('believe') A's message, then earning 500 only if the message equals the number actually assigned, or not follow and getting 150 points for sure. The strategy method is employed, asking A for her message for any number that might be assigned and asking B for his decision for any message that he might receive. After A and B make their decisions, the choices that result from the random number actually assigned to the pair are carried out. Note that this is a sender-receiver game with a high conflict of interests; the single equlibrium outcome is $m=6$ and 'not follow'.

In total 115 workers made decisions in part IV. Of these, $25 \%$ always reported truthfully while $50 \%$ always reported 6 . The responses of the remaining $25 \%$ were more mixed. These findings suggest that the fraction of lying averse subjects lies somewhere in between 0.25 and 0.5 , which seems to accord reasonably well with the findings in earlier (constant or high conflict) experiments. 
Yet these numbers do not match with our finding in the centralization game that honesty occurs in overall only $5 \%$ of the conflict cases (state 4). Apparently those who are averse to lying in the high conflict game of part IV have less of a problem with it in the centralization game where interests are often (but not always) aligned. ${ }^{19}$

A similar conclusion follows from correlating individual lying behavior in the two different parts. Let $m(i ; j)$ denote the message worker $j$ sends in part IV if the assigned number to the pair equals $i$. Then $L_{j}=\sum_{i=1}^{6}|m(i ; j)-i|$ measures the extent to which $j$ is prone to lying. If a worker always tells the truth $L_{j}=0$, whereas if a worker always reports 6 it holds that $L_{j}=15$. To arrive at an individual measure of lying behavior in the centralization game, we calculate the proportion of cases in which the worker lies out of all cases where conflict state 4 occurs. ${ }^{20}$ We consider two different versions. In the first lying in state 4 equals sending a message different than 'The state is 4' or 'I recommend Project A'. The Spearman correlation between this individual measure of lying behavior and $L_{j}$ appears to be small (0.08) and insignificant ( $p$-value of 0.47$) .{ }^{21}$ In the second measure lying in state 4 corresponds to sending a message that the state is 1,2 , or 3 . So recommending a project is not seen as lying. Also for this measure the Spearman correlation with $L_{j}$ is small (0.03) and insignificant (p-value of 0.81). ${ }^{22}$ Overall we therefore conclude that lying aversion is not the driver behind communication being more informative than predicted.

\subsubsection{Credulity}

A related explanation for more informative messages under centralization is that there is a significant fraction of credulous managers that believes any message received and act accordingly. We use the manager's following behavior in part IV to construct an individual measure of credulity.

\footnotetext{
${ }^{19}$ Also note that the lower bound of 0.25 lying averse workers would already be sufficient to support the Com-(3) outcome under strong alignment of interests, while the upper bound of 0.5 would not be sufficient to justify the same outcome under weak alignment of interests (cf. the discussion of the cutoff for fraction $\lambda$ in Subsection 2.3). Lying aversion thus cannot explain that even under weak interest alignment communication is more informative than predicted.

${ }^{20}$ Here we focus on parts I and II. For part III we get simliar results. Note, however, that in part III the group of workers playing the centralization game is not random. Only workers who are coupled with a manager who chose centralization are considered and the manager's decision is likely to be influenced by worker behavior in previous parts.

${ }^{21}$ An alternative measure of lying proneness follows from the 10 items taken from Lundquist et al (2009) included in the ex post questionnaire. Participants answered these items on a 1 (fully disagree) to 7 (fully agree) scale. Let $\widetilde{L}_{j}=\sum_{i=1}^{10} s(i)$, with $s(i)$ the score on item $i$. The Spearman rank correlation between $\widetilde{L}_{j}$ and $L_{j}$ is small $(0.11)$ and insignificant ( $p$-value of 0.23 ). This also holds for the correlation with the proportion of lying in the centralization game (0.13 with a $p$-value of 0.22$)$.

${ }^{22}$ The Spearman rank correlation between $\widetilde{L}_{j}$ (measure based on questionnaire data) and the second measure of proportion of lying in the centralization game is small and not significant (0.14 with a $p$-value of 0.17$)$.
} 
Let $r(i ; j) \in\{0,1\}$ denote whether (1) or not (0) manager $j$ follows message $i$. Then $C_{j}=$ $\sum_{i=1}^{6} r(i ; j) *(i-1)$ measure the extent to which $j$ is credulous. The idea behind this measure is that following after message 1 is not credulous. Besides in the true state 1 for truth-telling reasons, there are no other states in which subject $A$ has an incentive to lie and report 1 . This is different for message 6. Apart from when the state is indeed 6 (truth-telling), there are five other states where A has an incentive to lie and report 6 . Following after message 6 is thus particularly credulous. The overall measure $C_{j}$ weighs all the messages accordingly. It ranges from 0 (not credulous at all and only follow when message is one) to 15 (always follow, irrespective of A's message).

Of the 115 managers, almost $45 \%$ always decided to follow (so has $C_{j}=15$ ). $7 \%$ is not credulous at all and only follows after message 1 (i.e. $C_{j}=0$ ). For credulity to theoretically have an impact a fraction of at least $\frac{7}{11} \approx 64 \%$ is needed (cf. Subsection 2.3 ).

To further investigate to what extent credulity can explain manager behavior under centralization, we determine the proportion of cases in which the manager chooses to follow the worker's message. ${ }^{23}$ As previously discussed messages in M1 are sent only in state 1 and the manager best responds to these. Credulity is therefore best measured when the manager receives M2 or M3. We calculate the proportion of cases in which the manager chooses to implement project $B$ after receiving M2 or project $C$ after receiving M3. The Spearman correlation between this proportion and $C_{j}$ equals 0.15 and is marginally significant ( $p$-value equals 0.10). ${ }^{24} \mathrm{~A}$ similar conclusion follows if we look at factual statements and recommendations in isolation. The Spearman correlation between $C_{j}$ and the proportion of cases in which the manager chooses to follow the worker's message 'the state is 2 ' or 'the state is 3 ' is 0.07 and insignificant (p-value 0.46 ). For messages recommending $B$ or $C$ the correlation with $C_{j}$ equals 0.19 (p-value 0.08).

A potential drawback of our individual measure of credulity is that in many cases implementing project $B$ after M2 or project $C$ after M3 is the best response. To account for this we construct an alternative measure where we eliminate the cases where the manager is best responding. Hence, we calculate the proportion of cases in which the manager chooses to implement project $B(C)$ when the manager receives M2 (M3) and implementing project $B(C)$ is not a best response. The Spearman correlation between this proportion and $C_{j}$ is small (0.14) and insignificant ( $p$-value equals 0.13$)$. Taken together, also credulity cannot convincingly explain the more informative communication.

\footnotetext{
${ }^{23}$ The manager follows the worker's message if he considers statements about the state as true, follows the worker's recommendation and decides on the basis of the prior belief if the worker makes no recommendation.

${ }^{24}$ Again we focus on the data from Part I and II. The results for Part III data are similar, yet have to be interpreted with caution as only managers who at least once decided to keep the decision rights are included.
} 


\subsection{Mixed strategy communication equilibrium}

In the theoretical predictions we assumed that the worker and the manager employ a pure strategy. However, our findings show a pattern that might be in line with a mixed strategy equilibrium. By and large the data shows that the worker truthfully reports his type when the state is either 1, 2, or 3 , and pretends that his type is either 2 or 3 when the state equals 4 . The manager typically chooses $A$ after message M1, project $B$ if the message is M2, and switches between project $A$ and $C$ after M3. As the following proposition shows, this behavioral pattern can indeed be rationalized by mixed equilibrium play.

Proposition 4. If $\frac{66}{141} \leq q \leq \frac{33}{43}$ the communication game allows the following mixed strategy equilibrium, labelled Com-(3-mixed):

(W) The worker sends message $m_{1}$ when $t=1, m_{2}$ when $t=2, m_{3}$ if $t=3$, and employs a mixed strategy when $t=4$, sending $m_{2}$ with probability $\sigma_{2}=\frac{33-43 q}{33(1-q)}$ and $m_{3}$ with probability $\sigma_{3}=\frac{10 q}{33(1-q)} ;$

(M) The manager chooses $A$ after $m_{1}, B$ after $m_{2}$, and after $m_{3}$ she chooses $A$ with probability $\alpha_{A}=\frac{4}{11}$ and $C$ with probability $\alpha_{C}=\frac{7}{11}$.

When it exists, this equilibrium yields the manager more than optimal delegation.

A direct corollary of Proposition 4 is that, taking the Com-(3-mixed) equilibrium into account, optimal communication is weakly better than optimal delegation (cf. Proposition 3).

For $q=\frac{3}{4}$ the mixed strategy of the worker reduces to $\sigma_{2}=\frac{1}{11}$ and $\sigma_{3}=\frac{10}{11}$, while for $q=\frac{3}{5}$ this becomes $\sigma_{2}=\frac{5}{11}$ and $\sigma_{3}=\frac{6}{11}$. Managers should not change their behavior when moving from strong to weak alignment. Looking at actual behavior, under strong alignment workers choose $m_{2}$ in about $43 \%$ of the cases and $m_{3}$ in about $54 \%$ of the cases (cf. Table 5 ). Compared to the mixed equilibrium, they thus send message $m_{3}$ substantially less often than predicted (91\%). In case of weak interest alignment they choose $m_{3}$ in about $28 \%$ of the cases, again well below the $55 \%$ predicted. Comparative statics, however, are well in line with theoretical predictions: workers send $m_{3}$ substantially more often in the conflict state 4 when interests are strongly aligned than when they are weakly aligned. ${ }^{25}$

\footnotetext{
${ }^{25}$ We also observe mixed strategies at the individual level. Under strong (weak) interest alignment, about $35 \%$ $(60 \%)$ of the workers who observe state 4 more than once choose different messages in these instances, thus providing direct evidence for mixed strategy play. However, managers may effectively be exposed and thus perceive a mixed strategy more often, as they are each period randomly matched with a worker in the matching group. Around $60 \%$ $(80 \%)$ of the managers are exposed to a mixed strategy under strong (weak) alignment.
} 
For managers a similar picture emerges. In line with theoretical predictions, after message $m_{3}$ the manager either implements project $A$ or project $C$ in the majority of the cases. Only in $9 \%$ he chooses project $B$. In the strong alignment treatment managers choose project $A$ in about $42 \%$ of the cases and project $C$ in about 50\%. This compares reasonably well to the theoretical predictions of $36 \%$ and $64 \%$. Under weak interest alignment, managers choose $A$ in about $47 \%$ of the cases and $C$ in about $42 \%$. The manager's actual strategy is thus indeed fairly insensitive to variations in $q$, just as the mixed strategy equilibrium predicts. Qualitatively, results thus appear in line with the mixed strategy equilibrium. ${ }^{26}$

In Table 7 the actual payoffs are compared to the predicted payoffs in Com-(3-mixed) as well. These equal $80-\frac{260}{33} q$ for the manager and $80+\frac{40}{33} q$ for the worker. For managers the actual payoffs are not significantly different from the predicted payoffs. But workers earn significantly less than predicted in the mixed strategy equilibrium.

The fact that subjects are by and large able to coordinate on the mixed strategy communication equilibrium may also explain why managers frequently choose not to delegate. From Proposition 4 it follows that Com-(3-mixed) yields the manager more than any form of delegation does (on the domain of $q$ where the mixed equilibrium exists). A key feature of the mixed communication equilibrium driving this result is that the decision maker - here the manager - may optimally use a mixed strategy, such that the overall equilibrium relation between state and project choice can be stochastic. In contrast, after delegation the decision maker - in that case the worker will always employ a pure strategy, because he knows the state and in each state the different projects are strictly ordered for him. The equilibrium relation between state and project choice is then necessarily deterministic. This may be particularly restrictive when the optimal general mechanism is stochastic.

In a general mechanism the manager can commit to a probabilistic decision rule to choose project $a$ in state $t$ with probability $p_{t a}$. Using the revelation principle only direct mechanisms that induce truthtelling need to be considered to derive the optimal mechanism. It appears that in our setting the optimal mechanism is indeed stochastic for a large range of $q$ values.

Proposition 5. (i) The optimal deterministic mechanism corresponds to optimal delegation as in Proposition 1. (ii) The optimal general (stochastic) mechanism equals:

\footnotetext{
${ }^{26}$ Under strong (weak) interest alignment, around 55\% (38\%) of the managers respond differently to M3 on the multiple occasions they observe this message, thus pointing at individual level mixed play. Around $67 \%$ ( $80 \%$ ) of the workers are exposed to a mixed strategy under strong (weak) alignment, i.e. perceive mixed play by the manager.
} 
(ii.a) If $q \geq \frac{231}{271}:$ Del-(3) $=$ Com-(3);

(ii.b) If $\frac{11}{26} \leq q<\frac{231}{271}$ : The manager implements $A$ if $t=1, B$ if $t=2, A$ with probability $\frac{4}{11}$ and $C$ with probability $\frac{7}{11}$ if $t=3$, and $B$ if $t=4$;

(ii.c) If $q<\frac{11}{26}: \operatorname{Del}-(1)=\operatorname{Com}(1)$.

Note that the optimal mechanism in case (ii.b) corresponds to strategies (W) and (M) in Proposition 4 for $\sigma_{2}=1$. (Obviously, for $\sigma_{2}=1$ strategy (M) is no longer a best response to (W).) The worker therefore earns the same under the optimal mechanism as under Com-(3-mixed). The manager earns strictly more though.

The focus in the literature on communication and optimal delegation is predominantly on deterministic mechanisms. Kováč and Mylovanov (2009) theoretically explore a Crawford and Sobel type of framework in which sender (agent) and receiver (principal) both have quadratic preferences. They show that in this setting the optimal mechanism is necessarily deterministic if a certain regularity condition on the distribution of states and the conflict of interests holds. ${ }^{27}$ Alonso and Matouschek (2008) provide a specific example where this condition is violated and the optimal mechanism is stochastic. Departing from quadratic preferences, Kováč and Mylovanov (2009) provide another example in which the optimal mechanism is stochastic, the driving force being there that the principal's payoffs (an absolute value loss function) have less curvature than the agent's payoffs (a quadratic loss function). This enables the principal to use variance as an incentive device and thereby implement decisions that are closer to his ideal point than under a deterministic mechanism, without additional costs. Note that in our theoretical setup manager and worker are both assumed to be risk neutral with essentially symmetric payoffs. ${ }^{28}$

Our setup provides yet another specific example (but in an arguably natural setting) in which the optimal mechanism can be stochastic. Observations like these have typically been interpreted as having little practical relevance because, as noted by e.g. Alonso and Matouschek (2008) and Laffont and Martimort (2002, p. 67), the enforcement of random allocation rules may in practice be too problematic. Yet an interesting feature of our setting is that part of the gains of random allocation mechanisms are also captured by the Com-(3-mixed) equilibrium under centralization. This organizational structure may thus be an enforceable way to reap at least part of the benefits

\footnotetext{
${ }^{27}$ For the more specific case of a uniform prior and a constant conflict of interests across states, Goltsman et al (2009) provide an alternative proof that the optimal mechanism is deterministic.

${ }^{28}$ That is, only in the conflict state payoffs of projects $\mathrm{A}$ and $\mathrm{C}$ differ between manager and worker, but these are just the flip side of each other $((120,10)$ versus $(10,120))$.
} 
of stochastic mechanisms. Moreover, our experimental results reveal that this is not just a remote theoretical possibility. In the experiment subjects are by and large able to coordinate on the favorable mixed strategy outcome. ${ }^{29}$

\section{Conclusion}

In this paper we report the results from a laboratory experiment investigating a manager's decision whether to delegate decision authority to a better informed worker. The manager can keep authority and communicate with the worker, but divergent interests imply that workers communicate their information strategically. Alternatively, the manager can delegate the decision to the worker. A key characteristic of our setup is that the manager can restrict the agent's choice set when delegating authority ('restricted delegation'). Another key feature of our experimental design is that the worker's message should either be a factual statement about the state, or a recommendation about which project choice to make (or no recommendation at all). We find that, as expected, delegating managers put tighter restrictions if interests are less aligned. Workers send more informative messages under communication than predicted by the pure strategy equilibria. This finding neither appears to be driven by lying aversion of workers nor by credulity of managers. Qualitatively, our results are in line with a mixed strategy equilibrium under communication, which strictly outperforms optimal restricted delegation and is relatively close to the optimal stochastic mechanism in our setting.

A priori we expected 'over'-communication as compared to standard theory due to some subjects being lying averse. Although we do not find a big impact of lying aversion in our setting where interests are more often than not congruent, other cheap talk experiments like Cai and Wang (2006) do find over-communication if interests are always partly opposed. This seems to make communication rather than (restricted) delegation particularly attractive in these cases. At the same time it also suggests a potential way for managers to improve outcomes under delegation. If, after delegation, the worker is still required to send a message about the state - for instance

\footnotetext{
${ }^{29}$ It is straightforward to show that the optimal mechanism in game D of Lai and Lim (2012) is also stochastic, with action $a_{1}$ chosen for sure in the congruent state $t_{1}$ and actions $a_{2}$ and $a_{3}$ chosen with equal probabilities in conflict state $t_{2}$. Under this optimal mechanism both manager and worker earn more than under the unique babbling equilibrium under communication $((725,550) \succ(500,500))$. Qualitatively the data are in the direction of this outcome, see Figure 4 in Lai and Lim (2012). An alternative interpretation of their findings, therefore, is that managers (erronously) perceive the game as a repeated one, trying to build a reputation for committing to the optimal mechanism. In doing so, both players gain as compared to the uninformative babbling outcome. Alonso and Matouschek (2007) formally model such implicit commitment in an infinitely repeated cheap talk game and label it 'relational' delegation.
} 
as to justify or explain his choice of project - some workers may feel reluctant to lie and in turn also hesitant to choose the project that hurts the manager the most. Instead of restricting the worker's choice set to curb opportunistic behavior, it may in practice thus pay for the manager to let the worker feel accountable for his choices via a cheap talk justification. Investigating whether this would make unrestricted delegation more attractive for behavioral reasons seems an interesting avenue for future research. ${ }^{30}$

\section{References}

[1] Agastya, M., Bag, P.K. and I. Chakraborty, 2014, Communication and authority with a partially informed expert, Rand Journal of Economics 45, 176-197.

[2] Alonso, R. and N. Matouschek, 2007, Relational delegation, Rand Journal of Economics 38, 1070-1089.

[3] Alonso, R. and N. Matouschek, 2008, Optimal delegation, Review of Economic Studies 75, 259-293.

[4] Bartling, B., Fehr, E. and H. Herz, 2014. The intrinsic value of decision rights. Econometrica 82, 2005-2039.

[5] Bartling, B. and U. Fischbacher, 2012. Shifting the blame: On delegation and responsibility, Review of Economic Studies 79(1), 67-87.

[6] Blume, A., D.V. DeJong, Y.G. Kim, and G.B. Sprinkle, 1998. Experimental Evidence on the Evolution of Meaning of Messages in Sender-Receiver Games. American Economic Review, 88, $1323-1340$

[7] Blume, A., D.V. DeJong, Y.G. Kim, and G.B. Sprinkle, 2001. Evolution of Communication with Partial Common Interest. Games and Economic Behavior, 37, 79-120.

[8] Brandts, J. and D. Cooper, 2015. Centralized vs. decentralized management: An experimental study, working paper.

[9] Cai, H. and J.T-Y. Wang, 2006. Overcommunication in strategic information transmission games, Games and Economic Behavior 56, 7-36.

\footnotetext{
${ }^{30}$ See Xiao (2015) for a recent experiment investigating justification effects in a revised sender receiver game.
} 
[10] Charness, G. and M. Dufwenberg, 2010. Bare Promises: An Experiment, Economics Letters 107, 281-283.

[11] Coffman, L.C., 2011. Intermediation reduces punishment (and reward), American Economic Journal: Microeconomics 3(4): 77-106.

[12] Crawford,V, and J. Sobel, 1982. Strategic Information Transmission. Econometrica, 50, 14311451.

[13] Dessein, W., 2002. Authority and communication in organizations. Review of Economic Studies $69,811-838$.

[14] Dominguez-Martinez, S., Sloof, R. and F.A. von Siemens, 2014. Monitored by your friends, not your foes: Strategic ignorance and the delegation of real authority, Games and Economic Behavior, 85, 289-305.

[15] Evdokimov, P. and U. Gargagnini, 2015. Mend your speech a little: Authority, communication, and incentives to coordinate, working paper.

[16] Falk, A., and M. Kosfeld. 2006. The Hidden Costs of Control. American Economic Review, 96(5), 1611-1630.

[17] Farrell, J., 1993. Meaning and Credibility in Cheap-Talk Games. Games and Economic. Behavior, 5, 514-531

[18] Fehr, E., Herz, H. and T. Wilkening, 2013. The Lure of Authority: Motivation and Incentive Effects of Power. American Economic Review, 103(4), 1325-1359.

[19] Fershtman, C. and U. Gneezy, 2001. Strategic delegation: An experiment. Rand Journal of Economics, 32(2), 352-358.

[20] Filiz-Ozbay, E., and E.Y. Ozbay, 2007. Auctions with Anticipated Regret: Theory and Experiment. American Economic Review, 97(4), 1407-1418.

[21] Fischbacher, U., 2007. z-Tree: Zurich Toolbox for Ready-made Economic Experiments. Experimental Economics, 10(2), 171-178.

[22] Garfagnini, U., Ottaviani, M. and P.Norman Sørensen, 2014. Accept or reject? An organizational perspective, International Journal of Industrial Organization 34, 66-74. 
[23] Gneezy, U., 2005. Deception: The Role of Consequences. American Economic Review, 95(1), 384-394.

[24] Gneezy, U., B. Rockenbach, and M. Serra-Garcia, 2013. Measuring Lying Aversion. Journal of Economic Behavior and Organization, 93, 293-300.

[25] Goltsman, M., Hörner, J., Pavlov, G. and F. Squintani, 2009, Mediation, arbitration and negotiation, Journal of Economic Theory 144, 1397-1420.

[26] de Haan, Th., Offerman, Th. and R. Sloof, 2015. Money talks? An experimental investigation of cheap talk and burned money, International Economic Review 56, 1385-1426.

[27] Hamman, J.R., Loewenstein, G. and R.A. Weber, 2010. Self-interest through delegation: An additional rationale for the principal-agent relationship, American Economic Review, 100(4), 1826-1846.

[28] Hamman, J.R., and M.A. Martínez-Carrasco, 2015, Making the tough choices: Delegation and team selection in organizations, Working paper.

[29] Holmstrom, B., 1984. On the theory of delegation, in M. Boyer and R. Kihlstrom (eds.), Bayesian Models in Economic Theory, North-Holland, New York.

[30] Jensen, M.C. and W.H. Meckling, 1992. Specific and general knowledge and organizational structure, in L. Werin and H. Wijkander (eds.), Contract Economics, Blackwell, Oxford.

[31] Kartik, N., 2009. Strategic communication with lying costs. Review of Economic Studies, 76 (4), 1359-1395.

[32] Kováč, E. and T. Mylovanov, 2009, Stochastic mechanisms in settings without monetary transfers: The regular case, Journal of Economic Theory 144, 1373-1395.

[33] Laffont, J.-J. and D. Martimort, 2002. The theory of incentives: The principal-agent model, Princeton University Press.

[34] Lai, E.K. and W. Lim, 2012. Authority and communication in the laboratory. Games and Economic Behavior, 74, 541-560.

[35] Lundquist, T., T. Ellingsen, E. Gribbe, M. Johannesson, 2009. The aversion to lying. Journal of Economic Behavior and Organization, 70(1), 81-92. 
[36] Ottaviani, M., 2000. The economics of advice. mimeo.

[37] Pitchik, C., and A. Schotter, 1987. Honesty in a model of strategic information transmission. American Economic Review, 77 (5), 1032-1036.

[38] Oosterbeek, H., R. Sloof, and J. Sonnemans, 2011. Rent-seeking versus productive activities in a multi-task experiment. European Economic Review, 55 (5), 630-643.

[39] Owens, D., Grossman, Z. and R. Fackler, 2014. The control premium: A preference for payoff autonomy, American Economic Journal: Microeconomics 6(4): 138-161.

[40] Rush, A, Smirnov, V. and A. Wait, 2010. Communication Breakdown: Consultation or Delegation from an Expert with Uncertain Bias, The B.E. Journal of Theoretical Economics 10(1), $1-27$.

[41] Serra-Garcia, M., E. Van Damme, and J. Potters, 2011. Hiding an inconvenient truth: Lies and vagueness, Games and Economic Behavior, 73, 244-261.

[42] Sloof, R. and F. von Siemens, 2015. Decision initiation, decision implementation, and the allocation of decision rights, Tinbergen Institute Discussion Paper TI 2015-105/VII.

[43] Sobel, J., 2013. Giving and Receiving Advice, in: Advances in Economics and Econometrics, D. Acemoglu, M. Arellano and E. Dekel (eds.).

[44] Sobel, J., forthcoming. Ten possible experiments on communication and deception, Journal of Economic Behavior and Organization.

[45] Xiao, E., 2015, Justification, conformity and fairness, mimeo. 


\section{Appendix A: proofs}

Proof of Proposition 1. We first show that, given the structure of payoffs, Del-(3), Del-(2) and Del-(1) are the three relevant options to consider. Assuming that the worker always implements his most preferred project, it is never optimal for the manager to set $\Delta=\{B\}$, as this choice is dominated by $\Delta=\{A, B\}$. Clearly, $\Delta=\{C\}$ can never be optimal either, because project $C$ is dominated by project $B$ in expected payoff terms. Finally, $\Delta=\{A, C\}$ and $\Delta=\{B, C\}$ make no sense because full delegation would always yield the manager strictly more.

The optimal level of delegation thus follows from comparing the manager's expected payoffs in the three relevant cases. Under Del-(3) the manager earns $\frac{q}{3}(80+100+100)+(1-q) 10=$ $10+\frac{250}{3} q \equiv \Pi_{\text {Del-(3) }}$, under Del-(2) he gets $\frac{q}{3}(80+100+10)+(1-q) 80=80-\frac{50}{3} q \equiv \Pi_{\text {Del-(2) }}$ and under Del-(1) he earns $\frac{q}{3}(80)+(1-q) 120=120-\frac{280}{3} q \equiv \Pi_{\text {Del-(1) }}$. Comparing these payoffs, the thresholds for $q$ are obtained.

Proof of Proposition 2. We first show that the listed equilibrium partitions are the five relevant ones to consider, i.e. necessarily state 4 is in the same partition as state 3 .

Suppose that $\{4\}$ is a separate partition. The manager then chooses project $A$ in response. Given the structure of payoffs, there necessarily exists another partition that induces project $B$; this follows because states 1 through 3 are equally likely and project $B$ is the best response to any partition that contains state 2 . But then type $t=4$ would like to deviate to the message sent by the partition type $t=2$ is in. So $\{4\}$ cannot be separate. Next suppose type $t=4$ is not in the same partition as type $t=3$. If type $t=4$ is in the same partition as $t=1$ only, i.e. $\{1,4\}$, this partition induces project $A$. Again, as there is necessarily another partition that induces $B$ (viz. the one that contains $t=2$ ), type $t=4$ would like to deviate. In case of $\{2,4\}$, this partition induces $B$. But then the partition containing $t=3$ necessarily induces $C$ and type $t=4$ would like to switch. Finally, partition $\{1,2,4\}$ would induce either $A$ or $B$, while the remaining partition necessarily induces $C$. Again, type $t=4$ would like to switch. Taken together, type $t=4$ is necessarily in the same partition as $t=3$ is in.

Now consider the partition of case (a). Obviously, after $m_{\{1\}}$ the manager optimally chooses $A$ and after $m_{\{2\}}$ his best response is to choose $B$. If message $m_{\{3,4\}}$ would induce $A$, both $t=3$ and $t=4$ would like to deviate to $m_{\{2\}}$. So $m_{\{3,4\}}$ should either induce $B$ or $C$. The former would require that $\frac{q}{3} 10+(1-q) 80 \geq \max \left\{(1-q) 120, \frac{q}{3} 100+(1-q) 10\right\}$, i.e. both $q \geq \frac{12}{13}$ and $q \leq \frac{7}{10}$, which cannot hold. So the manager should necessarily optimally choose $C$ after $m_{\{3,4\}}$. This requires 
$\frac{q}{3} 100+(1-q) 10 \geq \max \left\{(1-q) 120, \frac{q}{3} 10+(1-q) 80\right\}$. Rewriting this gives $q \geq \max \left(\frac{7}{10}, \frac{33}{43}\right)=\frac{33}{43}$. Given that in that case each worker type gets the highest payoff possible, none of the types wants to deviate. In this equilibrium the manager's expected payoffs are equal to $\Pi_{\text {Del-(3) }}$ (see the proof of Proposition 1).

For the partition $\{1,3,4\}$ (cf. case (b)), project $A$ clearly yields the manager more than project $B$ does, so the relevant choice is between $A$ and $C$. If he would choose $A$, type $t=4$ would like to deviate to the message sent by $\{2\}$ (inducing $B$ ). So necessarily $C$ after $\{1,3,4\}$. This requires that $\frac{q}{3} 100+(1-q) 10 \geq \frac{q}{3} 80+(1-q) 120$. Rewriting gives $q \geq \frac{33}{35}$. In case (c) the manager's best response to $m_{\{1,2\}}$ is project $B$ necessarily. Given this, $m_{\{3,4\}}$ should not induce $A$, for otherwise both $t=3$ and $t=4$ would like to deviate. From case (a) above this requires $q \geq \frac{33}{43}$ necessarily.

Regarding the partition of case $(\mathrm{d})$, for $t \in\{2,3,4\}$ the manager strictly prefers $B$ over $C$. He prefers $B$ over $A$ if $\frac{q}{3}(100+10)+(1-q) 80 \geq(1-q) 120$, i.e. if $q \geq \frac{12}{23}$. The manager's expected payoffs in this equilibrium then equal $\Pi_{D e l-(2)}$. For $q<\frac{12}{23}$ the manager prefers $A$ over $B$ and we essentially obtain the same outcome as in case (e). (Although the manager could in this case distinguish between $\{1\}$ and $\{2,3,4\}$, this information is not influential as it does not affect the choice of project; we therefore do not list it as a separate case.) Finally, if the manager's choice of project is independent of the message received, worker types are indifferent between messages, and pooling on the same message thus is a best response (case (e)). Without additional information the manager prefers project $A$ over project $B$ whenever $q<\frac{4}{5}$. In that case he earns $\Pi_{D e l-(1)}$ in expecte payoffs.

Proof of Proposition 4. We show that the two strategies are best responses to each other. First suppose the worker uses strategy $(W)$. Receiving $m_{1}$ is then a clear signal of $t=1$, to which $A$ is indeed a best response. In case the manager receives $m_{2}$, the state equals either $t=2$ or $t=4$. For both these states $B$ is better for the manager than $C$ is, so the relevant choice is between $A$ and $B$. By Bayes' rule, the posterior belief that the state is $t=2$ equals $\beta_{2}=\operatorname{Pr}\left(t=2 \mid m_{2}\right)=\frac{q}{q+3(1-q) \sigma_{2}}$. Implementing project $A$ then yields $\beta_{2} \cdot 0+\left(1-\beta_{2}\right) \cdot 120$, while project B gives $\beta_{2} \cdot 100+\left(1-\beta_{2}\right) \cdot 80$. For $\beta_{2} \geq \frac{2}{7}$ the manager prefers project $B$. Rewriting this using the expression for $\beta_{2}$ gives $\sigma_{2} \leq \frac{5 q}{6(1-q)}$. For $\sigma_{2}=\frac{33-43 q}{33(1-q)}$ this reduces to $q \geq \frac{66}{141}$. For these values of $q$ the manager thus indeed prefers $B$. Finally, if the manager receives $m_{3}$, then he can infer that either $t=3$ or $t=4$. Let $\beta_{3}=\operatorname{Pr}\left(t=3 \mid m_{3}\right)=\frac{q}{q+3(1-q) \sigma_{3}}$. Implementing project $A$ yields $\beta_{3} \cdot 0+\left(1-\beta_{3}\right) \cdot 120$, project $B$ gives $\beta_{3} \cdot 10+\left(1-\beta_{3}\right) \cdot 80$, and project $C$ yields $\beta_{3} \cdot 100+\left(1-\beta_{3}\right) \cdot 10$. Compareing these payoffs 
it follows that $A$ yields the most if $\beta_{3}<\frac{11}{21}$, while project $C$ is the best choice for $\beta_{3}>\frac{11}{21}$. The manager is indifferent between $A$ and $C$ if $\beta_{3}=\frac{11}{21}$. Rewriting gives that $\sigma_{3}=\frac{10}{33} \frac{q}{1-q}$ is necessary for the manager to be willing to mix between $A$ and $C$. Now $\frac{10}{33} \frac{q}{1-q} \leq 1$ iff $q \leq \frac{33}{43}$, yielding the upper threshold on $q$.

Next suppose the manager uses strategy $(M)$. Obviously it is best for the worker to choose $m_{1}$ if $t=1$ and $m_{2}$ if $t=2$. In case the state equals $t=3$, the relevant choice is between $m_{2}$ and $m_{3}$. The former yields 10 with the latter gives $\alpha_{A} \cdot 0+\alpha_{C} \cdot 100=63 \frac{7}{11}$ for $\alpha_{C}=\frac{7}{11}$. Hence choosing $m_{3}$ is indeed better. In case $t=4$, the relevant choice is also between $m_{2}$ and $m_{3}$. The former yields the worker 80 , the latter gives $\alpha_{A} \cdot 10+\alpha_{C} \cdot 120=\frac{40+840}{11}=80$ as well. The worker is thus willing to mix between $m_{2}$ and $m_{3}$.

To prove the final claim, the expected payoffs for the manager in the mixed equilibrium are $80-\frac{260}{33} q \equiv \Pi_{\text {Com-(3-mixed })}$. This exceeds $\Pi_{\text {Del-(3) }}$ whenever $q \leq \frac{33}{43}$, it always exceeds $\Pi_{\text {Del-(2) }}$, and it exceeds $\Pi_{\text {Del-(1) }}$ whenever $q \geq \frac{66}{141}$.

Proof of Proposition 5. Let $p_{t a}$ be the probability that the manager implements project $a \in$ $\{A, B, C\}$ in state $t \in\{1,2,3,4\}$. The optimal general mechanism then follows from the following optimization program:

$$
\begin{aligned}
\max _{p_{t, a}} \Pi= & \frac{q}{3} \cdot\left(80 p_{1 A}+100 p_{2 B}+\left(10 p_{3 B}+100 p_{3 C}\right)\right) \\
& +(1-q) \cdot\left(120 p_{4 A}+80 p_{4 B}+10 p_{4 C}\right)
\end{aligned}
$$

subject to the following constraints: $0 \leq p_{t a} \leq 1 \forall t, a$

$$
\begin{array}{cc}
0 \leq p_{t a} \leq 1 \forall t, a & \text { (Prob_1) } \\
p_{t A}+p_{t B}+p_{t C}=1 \forall t & \text { (Prob_2) } \\
80 p_{1 A} \geq \max \left\{80 p_{2 A}, 80 p_{3 A}, 80 p_{4 A}\right\} & \text { (IC_1) } \\
100 p_{2 B} \geq \max \left\{100 p_{1 B}, 100 p_{3 B}, 100 p_{4 B}\right\} & \text { (IC_2) } \\
10 p_{3 B}+100 p_{3 C} \geq \max \left\{10 p_{1 B}+100 p_{1 C}, 10 p_{2 B}+100 p_{2 C}, 10 p_{4 B}+100 p_{4 C}\right\} & \text { (IC_3) }
\end{array}
$$




$$
10 p_{4 A}+80 p_{4 B}+120 p_{4 C} \geq \max \left\{\begin{array}{c}
10 p_{1 A}+80 p_{1 B}+120 p_{1 C}, \\
10 p_{2 A}+80 p_{2 B}+120 p_{2 C}, 10 p_{3 A}+80 p_{3 B}+120 p_{3 C}
\end{array}\right\}
$$

In case of a deterministic mechanism, condition (Prob_1) should be strengthened to $p_{t a} \in\{0,1\}$ for all $t, a$. We proceed in various steps, first proving two general features that hold in both optimal deterministic and optimal stochastic mechanisms alike.

(step 1) $p_{1 A}^{*}=1$ necessarily. To show this, note that $\Pi$ is increasing in $p_{1 A}$ and independent of $p_{1 B}$ and $p_{1 C}$. The l.h.s. of $\left(I C_{-} 1\right)$ is increasing in $p_{1 A}$, so more easily satisfied the higher $p_{1 A}$ is. Similarly so, both $\left(I C \_2\right)$ and $\left(I C \_3\right)$ are more easily satisfied the lower $p_{1 B}$ and $p_{1 C}$ are, i.e. the higher $p_{1 A}$ is. Also $\left(I C_{-} 4\right)$ is relaxed for higher $p_{1 A}$, as the first element in the $\max \{\}$ term on the r.h.s. necessarily gets smaller. Therefore the entire set of incentive constraints is relaxed the higher $p_{1 A}$ is. Given $p_{1 A}^{*}=1$, condition $\left(I C_{-} 1\right)$ is always satisfied and can be ignored from now on.

(step 2) $p_{2 C}^{*}=0$ necessarily. To see this, note that $\Pi$ is independent of $p_{2 C}$ (and $p_{2 A}$ ) and increasing in $p_{2 B}$. Condition $\left(I C \_2\right)$ is more easily satisfied when $p_{2 C}$ decreases, as this implies that either $p_{2 A}$ or $p_{2 B}$ gets higher. Also $\left(I C_{-} 3\right)$ and $\left(I C \_4\right)$ are relaxed for lower $p_{2 C}$, as the second elements in the $\max \{\}$ terms on the r.h.s. necessarily get smaller. If $p_{2 C}>0$ the manager would thus strictly benefit from shifting this probability mass to $p_{2 B}$ without harming feasibility.

(i) Consider deterministic mechanisms only, i.e. replace condition (Prob_1) with $p_{t a} \in\{0,1\}$ for all $t, a$. Given (step 1) and (step 2) above we consider the three relevant cases in turn.

(i.a) Suppose $p_{3 C}^{*}=1$. The r.h.s. of $\left(I C_{-} 4\right)$ then equals 120 and to satisfy this condition $p_{4 C}^{*}=1$ necessarily. The latter implies $p_{4 B}^{*}=0$ and thus that $\left(I C_{-} 2\right)$ is always satisfied. $p_{2 B}$ can then be chosen freely as to maximize $\Pi$, yielding $p_{2 B}^{*}=1$. The overall outcome $p_{1 A}^{*}=p_{2 B}^{*}=p_{3 C}^{*}=p_{4 C}^{*}=1$ corresponds to Del-(3) and yields the manager $\Pi_{D e l-(3)}$.

(i.b) Suppose $p_{3 C}^{*}=0$ and $p_{3 B}^{*}=1$. To satisfy $\left(I C \_2\right)$ then $p_{2 B}^{*}=1$ necessarily. Moreover, $p_{4 A}^{*}=1$ would then violate $\left(I C_{-} 4\right)$, so either $p_{4 B}^{*}=1$ or $p_{4 C}^{*}=1$ necessarily. The latter would violate $\left(I C \_3\right)$, hence $p_{4 B}^{*}=1$. The overall outcome $p_{1 A}^{*}=p_{2 B}^{*}=p_{3 B}^{*}=p_{4 B}^{*}=1$ corresponds to Del-(2) and yields the manager $\Pi_{D e l-(2)}$.

(i.c) Suppose $p_{3 C}^{*}=0$ and $p_{3 B}^{*}=0$. In that case $p_{3 A}^{*}=1$. To satisfy $\left(I C \_3\right)$ then $p_{2 A}^{*}=p_{4 A}^{*}=1$ necessarily. The overall outcome $p_{1 A}^{*}=p_{2 A}^{*}=p_{3 A}^{*}=p_{4 A}^{*}=1$ corresponds to Del-(1) with the worker always choosing $A$, yielding the manager $\Pi_{D e l-(1)}$. 
Comparing the expected payoffs of the manager in the three cases (cf. proof of Proposition 1), yields part (i) of the proposition.

(ii) Next consider general stochastic mechanisms, i.e. condition (Prob_1) has to be satisfied. Note that (step 1) and (step 2) continue to hold. We first prove two additional features of the optimal stochastic mechanim.

(step 3) $p_{3 B}^{*}=0$ necessarily. Suppose to the contrary that $p_{3 B}^{*}=p>0$. Then consider the alternative mechanism with $p_{3 B}^{\prime}=0, p_{3 A}^{\prime}=p_{3 A}^{*}+\frac{4}{11} p$ and $p_{3 C}^{\prime}=p_{3 C}^{*}+\frac{7}{11} p$. Given that $10 p_{3 B}^{\prime}+100 p_{3 C}^{\prime}=10 p_{3 B}^{*}+100 p_{3 C}^{*}+p\left(\frac{7}{11} \cdot 100-10\right)>10 p_{3 B}^{*}+100 p_{3 C}^{*}$, this leads to a strict increase in $\Pi$. Moreover, this alternative mechanism is still feasible as $\left(I C_{-} 2\right)$ is relaxed because the r.h.s. gets smaller, $\left(I C \_3\right)$ is relaxed because the l.h.s. gets larger, while the alternative mechanism is chosen such as to keep $\left(I C_{-} 4\right)$ unaffected.

(step 4) $7 p_{4 B}^{*}+11 p_{4 C}^{*}=11 p_{3 C}^{*}$ necessarily. This corresponds to $\left(I C \_4\right)$ being necessarily binding for mimicking type $t=3$. First suppose to the contrary that $\left(I C_{-} 4\right)$ does not bind at all. This can only happen when $p_{4 A}<1$. But then it is possible to increase $p_{4 A}$ at the margin, and thus decrease either $p_{4 B}$ or $p_{4 C}$ slightly. This would lead to a strict increase in $\Pi$ while still being feasible, because both $\left(I C_{-} 2\right)$ and $\left(I C_{-} 3\right)$ are relaxed. Thus $\left(I C_{-} 4\right)$ must bind necessarily. Next suppose it does not bind for mimicking type $t=3$. This can only happen when $p_{3 C}<1$. In that case $p_{3 C}$ can be increased at the margin to strictly improve $\Pi$, while relaxing $\left(I C \_3\right)$ and leaving $\left(I C \_2\right)$ unaffected. Hence $\left(I C \_4\right)$ binds for mimicking type $t=3$. From this it also follows that $\left(I C \_3\right)$ is always satisfied and can be ignored from now on.

Given (step 1) through (step 4) we consider the three relevant cases in turn.

(ii.a) Suppose $p_{3 C}^{*}=1$. Then the exact same reasoning as under (i.a) above gives the overall outcome $p_{1 A}^{*}=p_{2 B}^{*}=p_{3 C}^{*}=p_{4 C}^{*}=1$ (corresponding to Del-(3)), yielding the manager. $10+\frac{250}{3} q$ in expected payoffs.

(ii.b) Suppose $0<p_{3 C}^{*}<1$. Given that $\Pi$ is strictly increasing in $p_{2 B}$, either $p_{2 B}^{*}$ is such that $\left(I C_{-} 4\right)$ binds for mimicking type $t=2$ as well, or $p_{2 B}^{*}=1$. This implies $p_{2 B}^{*}=$ $\min \left\{p_{4 B}^{*}+\frac{11}{7} p_{4 C}^{*}, 1\right\}$. Together with $7 p_{4 B}^{*}+11 p_{4 C}^{*}=11 p_{3 C}^{*}$ from (step 4$)$ and $p_{4 A}^{*}=1-$ $p_{4 B}^{*}-p_{4 C}^{*}$ from (Prob_2), we can write $\Pi$ as a function of $p_{4 B}$ and $p_{4 C}$ only: $\Pi\left(p_{4 B}, p_{4 C}\right)=$ $\frac{q}{3} \cdot\left(80+100 \cdot \min \left\{p_{4 B}+\frac{11}{7} p_{4 C}, 1\right\}+100\left(\frac{7}{11} p_{4 B}+p_{4 C}\right)\right)+(1-q) \cdot\left(120-40 p_{4 B}-110 p_{4 C}\right)$. Note that $\Pi\left(p_{4 B}, p_{4 C}\right)$ is piecew-wise linear in $p_{4 B}$ and $p_{4 C}$. For $q<\frac{11}{26}$ it is strictly decreasing in both, implying $p_{4 A}^{*}=1$. But then by $7 p_{4 B}^{*}+11 p_{4 C}^{*}=11 p_{3 C}^{*}$ we must have $p_{3 C}^{*}=0$. Hence 
$0<p_{3 C}^{*}<1$ necessarily requires $q \geq \frac{11}{26}$. So assume $q$ to be such. Suppose the optimum is such that $p_{4 B}+\frac{11}{7} p_{4 C} \leq 1$. Then $\frac{\partial \Pi\left(p_{4 B}, p_{4 C}\right)}{\partial p_{4 B}}=\frac{100 q}{3} \cdot \frac{18}{11}-40(1-q)$ and $\frac{\partial \Pi\left(p_{4 B}, p_{4 C}\right)}{\partial p_{4 C}}=$ $\frac{100 q}{3} \cdot \frac{18}{7}-110(1-q)$. For $q<\frac{1617}{2337} \simeq 0.69$, it holds that $\frac{\partial \Pi\left(p_{4 B}, p_{4 C}\right)}{\partial p_{4 B}}>\frac{\partial \Pi\left(p_{4 B}, p_{4 C}\right)}{\partial p_{4 C}}>0$, which implies $p_{4 B}^{*}=1$. For $q>\frac{1617}{2337}$, it holds that $\frac{\partial \Pi\left(p_{4 B}, p_{4 C}\right)}{\partial p_{4 C}}>\frac{\partial \Pi\left(p_{4 B}, p_{4 C}\right)}{\partial p_{4 B}}>0$, which would imply $p_{4 C}^{*}=1$ and $p_{4 B}+\frac{11}{7} p_{4 C} \leq 1$ does not hold. Next suppose the optimum is such that $p_{4 B}+\frac{11}{7} p_{4 C} \geq 1$. Then $\frac{\partial \Pi\left(p_{4 B}, p_{4 C}\right)}{\partial p_{4 B}}=\frac{100 q}{3} \cdot \frac{7}{11}-40(1-q)$ and $\frac{\partial \Pi\left(p_{4 B}, p_{4 C}\right)}{\partial p_{4 C}}=\frac{100 q}{3}-110(1-q)$. For $q<\frac{231}{271} \simeq 0.85$, it holds that $\frac{\partial \Pi\left(p_{4 B}, p_{4 C}\right)}{\partial p_{4 B}}>\frac{\partial \Pi\left(p_{4 B}, p_{4 C}\right)}{\partial p_{4 C}}>0$, which implies $p_{4 B}^{*}=1$. For $q>\frac{231}{271}$, it holds that $\frac{\partial \Pi\left(p_{4 B}, p_{4 C}\right)}{\partial p_{4 C}}>\frac{\partial \Pi\left(p_{4 B}, p_{4 C}\right)}{\partial p_{4 B}}>0$, which would imply $p_{4 C}^{*}=1$. But then by $7 p_{4 B}^{*}+11 p_{4 C}^{*}=11 p_{3 C}^{*}$ we must have $p_{3 C}^{*}=1$. Hence $0<p_{3 C}^{*}<1$ necessarily requires $q \leq \frac{231}{273}$. Taken together, $0<p_{3 C}^{*}<1$ implies that $p_{4 B}^{*}=1=p_{2 B}^{*}$ necessarily and thus $p_{3 C}^{*}=\frac{7}{11}$ from (step 4). The overall outcome thus equals: $p_{1 A}^{*}=p_{2 B}^{*}=p_{4 B}^{*}=1, p_{3 A}^{*}=\frac{4}{11}$ and $p_{3 C}^{*}=\frac{7}{11}$. This yields the manager $80-\frac{260}{33} q$.

(ii.c) Suppose $p_{3 C}^{*}=0$. From (step 3) we have $p_{3 B}^{*}=0$ and the exact same reasoning as under (i.c) applies; the overall outcome equals $p_{1 A}^{*}=p_{2 A}^{*}=p_{3 A}^{*}=p_{4 A}^{*}=1$ (i.e. Del-(1)), yielding the manager $120-\frac{280}{3} q$ in expected payoffs.

Comparing the expected payoffs of the manager in the three cases, yields part (ii) of the proposition.

\section{Appendix B: sample instructions [treatment CD75]}

\section{Instructions Experiment}

\section{General Information}

Thank you for participating in this experiment. The amount of money you earn depends upon the decisions you and the other participants make. Your earnings are given in points. The experiment consists of four parts. Your overall earnings equal the sum of your points in each part. The conversion rate is 2.5 points for 1 eurocent, so 250 points corresponds with 1 euro. We will pay out your overall earnings in cash after you have completed the entire experiment and filled out a final questionnaire. We ensure that your final earnings remain confidential: no other participant from the experiment will learn your final earnings. 
This (two-sided) sheet contains the instructions for part one of the experiment. Instructions for the next part follow after this part has been completed (and so on). Please do not communicate with other participants during the experiment. If you have a question, please raise your hand. The experimenter will then come to your table to answer your question in private.

\section{Instructions part I}

There are two types of participants: managers and workers. One half of the participants will be managers, and the remaining half will be workers. You will be randomly assigned one of these roles. Which role you have, you will learn at the start of this part. You will keep the same role in parts II and III. In part one 20 project implementation decisions have to be made. In every project implementation decision (period), manager and worker face three projects $(\mathbf{A}, \mathbf{B}, \mathbf{C})$ that can be implemented. These projects differ in the points that they yield manager and worker upon implementation. The points belonging to a given project depend on the state of the world. There are four possible states $(\mathbf{1 , 2 , 3 , 4 )}$. The following table presents the points the different projects yield manager and worker in the different states:

\begin{tabular}{|l|cc|cc|cc|}
\hline & \multicolumn{2}{|c|}{ Project A } & \multicolumn{2}{c|}{ Project B } & \multicolumn{2}{c|}{ Project C } \\
& Manager & Worker & Manager & Worker & Manager & Worker \\
\hline State 1 & 80 & 80 & 0 & 0 & 0 & 0 \\
State 2 & 0 & 0 & 100 & 100 & 0 & 0 \\
State 3 & 0 & 0 & 10 & 10 & 100 & 100 \\
State 4 & 120 & 10 & 80 & 80 & 10 & 120 \\
\hline
\end{tabular}

At the beginning of each period the computer determines the state. The four states are equally likely. That is, with probability $25 \%$ the state is 1 , with probability $25 \%$ the state is 2 , with probability $25 \%$ the state is 3 , and with probability $25 \%$ the state is 4 .

As explained above, Part I consists of 20 periods. In each period, one manager and one worker are randomly paired. You are never paired with the same other participant twice in a row. You cannot predict when you will be paired with the same other participant again.

At the beginning of each period the worker learns the state. The manager decides which project (either A, B, or C) will be implemented. Before the manager does so, the worker sends a message to the manager. The manager only learns the actual state at the end of the period, after the project has been implemented. 


\section{Sequence of Actions}

The precise timing within each period is as follows. There are two phases.

\section{Phase 1}

The worker learns the state and sends one of the following eight messages to the manager:

The state is 1

The state is 2

The state is 3

The state is 4

I recommend project A

I recommend project $\mathrm{B}$

I recommend project $\mathrm{C}$

I make no recommendation

The set of available messages does not depend on the actual state; so irrespective of the actual state, the worker can always choose one of the above eight messages.

\section{Phase 2}

The manager observes the worker's message (but not the actual state) and decides which project to implement: either A, B, or C.

Upon completion of phase 2 both manager and worker are informed about the outcome in that period.

\section{Payoffs}

The number of points earned by manager and worker respectively are their points from the implemented project; see the table on the other side of the sheet.

Your overall payoff from this part is the sum of points earned in the 20 periods.

\section{Instructions Part II}

This part of the experiment also consists of 20 periods. As compared to part I the main difference is that now the worker takes the implementation decision in each period. Before s/he does so, the manager may restrict the set of projects that the worker can choose from. 
As before, in each period one manager and one worker are randomly paired. You are never paired with the same other participant twice in a row. You cannot predict when you will be paired with the same other participant again.

Like in part I the worker learns the state (the four different states occur with the same probabilities

as in part I). Before the worker decides which project to implement, the manager decides which projects the worker is allowed to choose for implementation. The manager only learns the state at the end of the period, after the project has been implemented.

\section{Sequence of Actions}

The precise timing within each period is as follows. There are two phases.

Phase 1

The manager selects the projects that the worker is allowed to choose for implementation.

Phase 2

The worker learns the state and the set of projects from which s/he is allowed to choose, and decides which of these projects to implement. (If the manager only allows one project, this becomes a trivial choice.)

Upon completion of phase 2 both manager and worker are informed about the outcome in that period.

\section{Payoffs}

The number of points earned by manager and worker respectively are their points from the implemented project. The same table as in part I applies.

Your overall payoff from this part is the sum of points earned in the 20 periods.

\section{Instructions Part III}

This part of the experiment combines the previous two parts and consists of 20 project implementation decisions (periods). At the beginning of each period the manager first decides whether s/he will take the implementation decision her- or himself or whether s/he delegates the implementation decision to the worker. The former corresponds with the decision structure of part I, the latter with the decision structure of part II. 
As in the previous parts, in each period one manager and one worker are randomly paired. You are never paired with the same other participant twice in a row. You cannot predict when you will be paired with the same other participant again.

\section{Sequence of Actions}

The precise timing within each period is as follows. There are three phases.

Phase 0

The manager chooses between taking the project implementation decision her- or himself, Decision Manager, and delegation of this decision to the worker, Decision Worker.

Phases 1 and 2

The structure of phases 1 and 2 depends on the manager's choice in phase 0:

\begin{tabular}{|l|l|l|}
\hline \multicolumn{1}{|c|}{$\begin{array}{c}\text { Decision Manager } \\
\text { (phases } 1 \text { and } 2 \text { are as in part I) }\end{array}$} & \multicolumn{1}{c|}{\begin{tabular}{c}
\multicolumn{1}{c|}{ Decision Worker } \\
(phases 1 and 2 are as in part II)
\end{tabular}} \\
\hline Phase 1 & $\begin{array}{l}\text { The worker learns the state and sends a } \\
\text { message(out of the eight messages } \\
\text { possible) to the manager; }\end{array}$ & $\begin{array}{l}\text { The manager selects the projects that the } \\
\text { worker is allowed to choose for } \\
\text { implementation }\end{array}$ \\
$\begin{array}{l}\text { The manager observes the worker's } \\
\text { message (but not the actual state) and } \\
\text { decides which project to implement }\end{array}$ & $\begin{array}{l}\text { The worker learns the state and the set of } \\
\text { projects from which s/he is allowed to } \\
\text { choose, and decides which of these } \\
\text { projects to implement }\end{array}$ \\
\hline
\end{tabular}

\section{Payoffs}

The number of points earned by manager and worker respectively are their points from the implemented project. The same payoff table as in parts I and II applies. Your overall payoff from this part is the sum of points earned in the 20 periods. 


\section{Overview parts I and II}

Before the start of the first period in part III, you will get a history overview of your own outcomes in parts I and II.

\section{Instructions Part IV}

The final part has a structure that differs from the previous parts. It consists of only one period. You are randomly paired with one other participant, taking either the role of participant A or participant B.

The computer will randomly assigns one of the numbers $1,2,3,4,5$, or 6 to each pair. Based on the assigned number, participant A sends a message about that number to Participant B. Participant B observes the message sent by Participant A, but not the number actually assigned by the computer, and decides whether or not to follow the message of Participant A.

Both participants A and B indicate their choices for each situation that may occur. In particular, for every possible number assigned to the pair, participant A has to formulate a message to participant B about the assigned number. The message does not have to contain the actual assigned number:

\begin{tabular}{|l|c|c|c|c|c|c|}
\hline If the assigned number is & 1 & 2 & 3 & 4 & 5 & 6 \\
\hline $\begin{array}{l}\text { then your message to participant B is: } \\
\text { The assigned number is }\end{array}$ & --- & --- & --- & --- & --- & --- \\
\hline
\end{tabular}

Before participant $\mathrm{B}$ receives the actual message of participant $\mathrm{A}, \mathrm{s} /$ he has to decide for all possible messages whether or not $\mathrm{s} /$ he will follow the message:

\begin{tabular}{|l|l|}
\hline If participant A sends the message & Then your decision is: \\
\hline "The assigned number is 1" & to follow O O not to follow \\
\hline "The assigned number is 2" & to follow O O not to follow \\
\hline "The assigned number is 3" & to follow O O not to follow \\
\hline "The assigned number is 4" & to follow O O not to follow \\
\hline "The assigned number is 5" & to follow O O not to follow \\
\hline "The assigned number is 6" & to follow O O not to follow \\
\hline
\end{tabular}

After A and B have made their decisions, the choices that result from the number actually assigned to the pair are carried out. That is, given the assigned number, the corresponding message of 
participant $\mathrm{A}$ is sent to participant $\mathrm{B}$ and the decision of participant $\mathrm{B}$ corresponding to this message is carried out.

\section{Payoffs}

Participant A receives 500 points plus 100 times the number sent in the message to Participant B. This means that if Participant A sends the message that the assigned number is 1 , then her/his payoff equals 600 points; if Participant A sends the message that the number is 2, then her/his payoff equals 700 points; and so on.

The payoff of participant B depends on whether s/he decides to follow Participant A or not and on whether the message of Participant A contains the actual assigned number to the pair.

If Participant B decides to follow, then Participant B receives 500 points if the message of Participant A contains the actual assigned number to the pair. Otherwise Participant B receives zero. This means that if Participant B decides to follow and the actual assigned number to the group is 1 and the reported number in the message of Participant A is 1, then Participant B gets 500 points; however, if Participant B decides to follow and the actual assigned number to the group is 1 and the reported number in the message of Participant A is 2, then Participant B earns zero points; and so on.

If Participant B decides not to follow, then Participant B receives 150 points. 